\title{
Gran Paradiso - Mont Blanc: Prähistorische und historische Gletscherstände
}

\author{
Von Adolf Zienert, Heidelberg \\ Mit 11 Abildungen im Text
}

$\mathrm{Z}$ us a m me $\mathrm{n} f$ a s s ung. Das Untersuchungsgebiet umfaßt die westlichen und südlichen Teile der Aosta-Region vom Hauptkamm der M. Blanc-Gruppe bis zum nach N entwässernden Teil der Paradiso-Gruppe. Die Untergrenze bildet das Moränengebiet von Aymavilles-Sarre, etwa $5 \mathrm{~km}$ westlich Aosta. Verfolgt werden die durch Moränen gekennzeichneten Gletscherstände vom "Aymavilles“ (= „Gschnitz") bis zur Gegenwart und die Vermessungsergebnisse ab 1929-31 (Karte $1: 25$ 000); die letzten genaueren Höhenwerte liegen für 1950-53 vor. Moränen aus historischer Zeit gibt es etwa von $1600,1680,1770,1820,1860$ und 1920. Nur wenige Gletscher weisen deutliche noch jüngere Moränen auf. Tiefliegende Moränen aus dem 17. und 18. Jahrh. lassen sich leider nicht immer eindeutig einem der drei Stände dieses Zeitraumes zuordnen. Innerhalb der bisher als "Courmayeur" bezeichneten und dem "Daun“ gleichgesetzten Moränen kann man deutlich zwei Gruppen unterscheiden. Es wird deshalb vorgeschlagen, die Bezeichnung „Courmayeur" durch zwei neue Lokalnamen aus der Umgebung von Cogne, "Epinel“ (= "Daun“) und "Valnontey" (= „Egesen“), zu ersetzen. Zum Schluß wird versucht, die Geschichte der Gletscher im Untersuchungsgebiet kurz darzustellen.

$\mathrm{S} \mathrm{u} \mathrm{m} \mathrm{m} \mathrm{a} \mathrm{ry.} \mathrm{The} \mathrm{so-called} \mathrm{Aosta-Region} \mathrm{(named} \mathrm{after} \mathrm{its} \mathrm{capital} \mathrm{Aosta} \mathrm{and} \mathrm{the} \mathrm{main} \mathrm{valley}$ the Valle d'Aosta) lies in the northwesternmost part of Italy. In its western and southern secondary valleys (the northern are excluded here) there are a lot of glaciers from the southeastern side of the chain of Mont Blanc to the north-side of the group of Gran Paradiso (Italians are numbering 140 glaciers). This paper deals with the moraines of the more significant of these glaciers from prehistoric times to the present. The following stadials can be distinguished: Aymavilles-Gschnitz, three miles to the west of Aosta, Epinel - Daun, usually at about the middle of the secondary valleys, and Valnontey - Egesen, occasionally even in tertiary valleys. Out of historic times there can be found moraines from about 1600,1680,1770,1820, 1860 and 1920. Measurements, especially of the lowest points the ice was then filling, have been made by the Italians for cartographic reasons in 1929-31 and continued for hydroelectric developments (latest figures published: 1950-53). All these moraines and datas are discussed in this context to obtain the elements of glacial history.

$\mathrm{R}$ ésumé. La région étudiée embrasse les parties occidentales et méridionales de la vallée d'Aoste depuis le massif du Mont Blanc jusqu'à la partie du Grand Paradis, draînant ses eaux vers le nord. La limite inférieure est déterminée par les formations stadiaires d'AymavillesSarre, situé à environ $5 \mathrm{~km}$ à l'ouest d'Aoste. L'auteur s'est proposé d'étudier d'une part les différents stadials (et stades) glaciaires indiqués par les moraines depuis l' "Aymavilles" (="Gschnitz") jusqu'à l'époque actuelle et d'autre part les relevés topographiques depuis 19291931 (carte 1:25 000); les dernières cotes de hauteur précises datent de 1950-1953. On peut dénombrer, d'époque historique, des moraines en 1600, 1680,1770, 1820, 1860 et 1920. Peu de glaciers présentent des moraines plus récentes. Les moraines profondes du $17 \mathrm{e}$ ou du $18 \mathrm{e}$ siècle ne se laissent pas toujours facilement inclure dans un de trois stades de cette épogue. On peut gistinguer, dans les moraines désignées jusqu'ici du nom de "Courmayeur" et assimilées au "Daun", deux groupes. L'auteur propose donc de substituer à la dénomination "Courmayeur" deux noms des environs de Cogne: "Epinel" (="Daun") et "Valnontey" (="Egesen"). L'auteur s'efforce, pour ter miner, de brosser un court tableau de l'histoire des glaciers dans la région incriminée.

\section{Einleitung}

Die Erforschung der Gletscher des Untersuchungsgebietes (vgl. die Übersichtskarte Abb. 1), vor allem derjenigen der M. Blanc-Gruppe, begann sehr früh. Eine große Rolle spielte dabei das Hinunterreichen einiger Gletscher bis in die Täler, des Brenva-Gletschers sogar bis in das Kulturland. Zusammenstellungen der mündlichen und schriftlichen Uberlieferung über die Geschichte der Gletscher seit dem 15. Jh. und über die Eisrandlagen vom 19. Jh. an gibt es deshalb mehrere.

An Sammlungen vor allem der his to r is che n Quelle n wären zu nennen: Für das M. Blanc-Gebiet allgemein SACCO 1919 und 1942, dazu für den Brenva-Gletscher 
Marengo 1881, Porro 1903 und Valbusa 1927; für den Rutor-Gletscher Baretti 1880 a und SACCO 1917 a; für das Paradiso-Gebiet SACco 1921 und 1923, dazu Porro 1903 (Tráio, Valnontey, Bardoney).

$\mathrm{Zu}$ den $\mathrm{E}$ is $\mathrm{r}$ andlage $\mathrm{n}$ werden in jedem Band des Bollettino del Comitato Glaciologico Italiano die Ergebnisse der neuesten Vermessungen veröffentlicht, z. T. mit Kartenskizzen oder sogar mit Spezialkarten.

Den Stand von 1929-31 (je nach Vermessungsjahr), im M. Blanc-Gebiet zum großen Teil zusätzlich noch denjenigen von 1947 (Neuvermessung), kann man den topographischen Karten 1: 25000 des Istituto Geografico Militare (IGM) entnehmen.

Zusammenfassende Angaben über den Gletscherrückgang von 1929/31 bis 1952 enthält die Arbeit von VANNi, Origlia \& DE Gemini 1953. Detailliertere Angaben für längere oder zumindest mehrjährige Zeiträume und meist auch Karten dazu enthalten vor allem: Marengo 1881, Silvestri 1925, Valbusa 1927 und Capello 1941 (alle Brenva), Capello 1940 (Pré-de-Bar, Triolet, Lex-Blanche), Peretti 1934 und SAcco 1934 a (Rutor).

Außerdem sind in den meisten Veröffentlichungen Photos enthalten, im allgemeinen mit Angaben über das Jahr oder sogar den Tag der Aufnahme.

Zur Morphometrie gibt es recht interessante, weitgehend statistisch arbeitende Untersuchungen von CAPELLO über die Gletscher der M. Blanc-Gruppe - Stand 1929 (1936 b) und über die Gletscher im Val di Rhêmes (1948).

Wesentlich weniger gutes Material findet man über die Mor än en großer Teile des Untersuchungsgebietes. Zwar hat $\mathrm{S}_{\mathrm{ACCO}}$ über fast alle Gletschergebiete vom M. Blanc bis zum Paradiso in verschiedenen Arbeiten Karten veröffentlicht (zunächst in 1:100000, später in 1:50000, schließlich in 1:25000; über den Miage-Gletscher bereits 1917 sogar in $1: 10000$ ), aber leider, und er gibt es mehrfach selbst $\mathrm{zu}$, sind die Karten alle mehr oder weniger schematisch gehalten, selbst die oben erwähnte Karte 1:10000 (dazu z. B. SAcco 1942, S. 4, die letzten Zeilen von Absatz 3). Wirklich gut sind in den älteren Arbeiten nur die Karten in BARETTI 1880 b (Miage 1:10000, mit Darstellung selbst der Vegetationsdecke älterer Moränenteile) und in MARENGo 1881 (Brenva 1:20000, ebenfalls mit Vegetationsangaben); dazu käme noch die Arbeit von Peretri 1937. Viel mehr oder zumindest ebenso viel wie den meisten übrigen Karten, vor allem denjenigen von SACCO, kann man bei entsprechender Geländeerfahrung einfach den topogr. Karten $1: 25000$ des IGM entnehmen. Es gibt aber eine große, neuere Ausnahme: Beschel 1958 bietet für die historischen Moränen in den Hochlagen (leider aber auch nur für diese) des Paradiso-Hauptgebietes mit Hilfe der Lichenometrie ausgezeichnete Ergebnisse.

\section{Die Gletscherstände}

Bei den Angaben für 1929 und später in den folgenden Tabellen handelt es sich vorwiegend um Eisrandlagen. Die Angaben für die Zeit vor 1929 beziehen sich auf im Gelände sichtbare Anhaltspunkte für die damalige Lage der Gletscherenden, vor allem natürlich auf Moränen. Angeführt wird jeweils die Meereshöhe („m $\mathrm{MH}^{“)}$ der tiefstgelegenen Stelle, sowie die horizontale und die vertikale Komponente der Abtsände zwischen den durch die Höhenwerte gekennzeichneten Punkten. Selbstverständlich sind die Bezeichnungen 1920, 1860 usw. nur als Sammelbezeichnungen mit einer Fehlergrenze von einigen Jahren zu verstehen.

Die Zahlen für 1952 stammen etwa zur Hälfte aus der Zusammenstellung von VANNI etc. im Boll. Comit. glac. ital. 1953 (dort "Quota minimale"). Leider waren die übrigen und deshalb hier nicht aufgeführten Höhenzahlen aus jener Arbeit offensichtlich falsch. Die Gletscher hätten dazu in der Zeit nach 1929/31 stark vor- statt zurückgehen 
müssen, oder die Zahlen stammten von 1947 und 1929 (ohne Vermerk dabei), oder es waren z. B. die kürzeren Zipfel der Gletscher als am tiefsten hinunterreichend gezählt. Ein großer Teil der übriggebliebenen Zahlen mußte leider zumindest mit „etwa“ oder „?“ versehen werden (z. B. die vollen Hunderter). Soweit möglich, wurden bessere Werte aus anderen Publikationen eingesetzt. Stammen diese aus anderen Jahren, so wird die entsprechende Jahreszahl (statt 1952) angegeben.

Die Zahlen für 1947 aus dem Val Ferret entstammen der Neuausgabe des entsprechenden Blattes der Karte 1:25000 des IGM bzw. der schönen, mehrfarbigen Carta Turistica del M. Bianco 1:25000 des IGM. Leider wurden 1947 die unteren Teile der Gletscher im Val de la Lex Blanche und im Val Veni nicht neu vermessen.

Die Angaben für 1929/31+33 wurden den Karten 1:25000 des IGM entnommen. In den folgenden Listen sind jeweils die Vermessungsjahre angegeben.

Die Höhenzahlen für 1920 u n d f r üh e r wurden ebenfalls den Karten 1:25000 des IGM entnommen, aber erst nach Eintragung der Gletscherstände durch den Verfasser. Dabei wurden alle in der Literatur (vor allem BEschel 1958) und im Rahmen der Geländearbeit entwickelten Kriterien zur Bestimmung der betreffenden Lage herangezogen. (siehe Abschnitt: die Moränen). Soweit keine einigermaßen sicheren Angaben aufzutreiben waren (für 1929 und später), oder die Lage der Gletscherenden aus z. T. sehr verschiede-

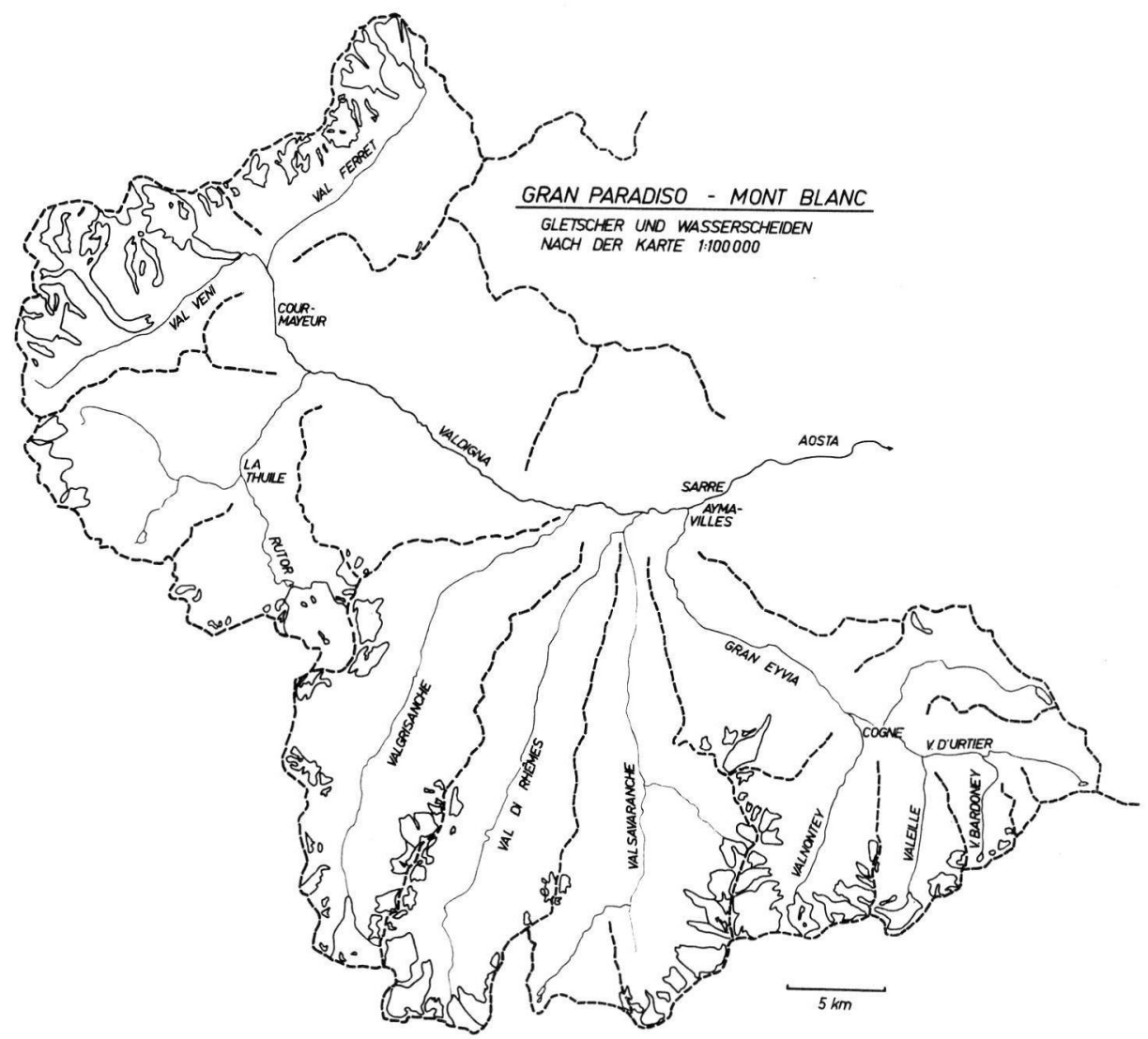

Abb. 1. Ubersichtskarte des Gebietes von der Mont-Blanc-Gruppe (NW) bis zum Gran-ParadisoNationalpark (SO). 
nen Gründen nicht wenigstens mit einiger Wahrscheinlichkeit festzustellen war, wurde „?" gesetzt.

In den folgenden Aufstellungen steht (nach dem Namen des Tales) jeweils zuerst der Name des Gletschers, mit zwei Ausnahmen (Eaux-Rouges und Bardoney) nach den Karten $1: 25000$ des IGM, und dahinter in Klammer die Nr. nach VANNI etc. 1953. „c.“ vor einer Zahl steht für etwa (circa). „HD“ bedeutet Horizontaldifferenz, „VD“ Vertikaldifferenz. Als Basispunkt für die Berechnung von HD und VD wurde jeweils der durch die fettgedruckte Höhenzahl gekennzeichnete Punkt benutzt. Die Genauigkeit dieser Differenzwerte hängt natürlich von der Genauigkeit der zur Verfügung stehenden Grundwerte ab, eine Kennzeichnung der Genauigkeit durch „c.“ oder eine ähnliche Bezeichnung erfolgt deshalb nicht. „(B)" hinter einer Höhenzahl heißt: nach BEsches 1958 oder zumindest gleicher Befund hier und dort, „Fp“ Gletscherende auf Felsplatten geringer bis mittlerer Neigung, „F“ in Steilstufen oder auf steilen Felspartien ohne Halt für Moränenwälle, „T“ Unterkante im Toteis. „Valnontey“ und „Epinel“ (abgekürzt „Va.“ und „Ep.“) werden vorläufig die beiden in fast allen Tälern verfolgbaren prähistorischen Gletscherstände genannt.

$\mathrm{m} \mathrm{MH}$

\section{Vallone d'Urtier}

Peradzá (131)

$\begin{array}{ll}1952 & \text { c. } 2900 \text { ?, Fp } \\ 1933 & \mathbf{2 8 3 2} \mathrm{Fp} \\ 1920 & \mathrm{Fp} \\ 1860 & \mathrm{Fp} \\ 1820 & \mathrm{Fp} \\ 17 / 18 . \mathrm{Jh} & \mathrm{Fp} \\ \text { Va. } & \text { c. } 23701 \text { 1) } \\ \text { Ep. } & \text { c. } 1980 \text { (A. Crêt) }\end{array}$

1) Gletscherende sehr breit, Blockstreu.

\section{Vallone di Bardoney}

Eaux Rouges (130)

\begin{tabular}{|c|c|}
\hline 1952 & c. 2800 \\
\hline 1931 & 2736 \\
\hline 1920 & c. 2730 \\
\hline 1860 & $\mathrm{~F}$ \\
\hline $\begin{array}{l}1820 \\
17 / 18 \text {. Jh. }\end{array}$ & $\begin{array}{l}F \\
F\end{array}$ \\
\hline
\end{tabular}

in Va. und Ep. unselbständig (s. Bardoney)

L a vin a (129) N-/S-Teil

$\begin{array}{llll}195,2 & \text { c. } 2800 & \text { c. } 2700 \\ 1931 & 2749 & \text { (schmal) } / & 2673 \mathrm{~F} \\ 1920 & \text { c. } 2740 \text { (breit) } / & \text { F } \\ 1860 & \text { c. } 2660 & \text { unselbständig } \\ 1820 & \text { F } & \\ 17 / 18 . & \text { Jh } & \text { F } & \end{array}$

17/18. Jh.

$\mathrm{F}$

in Va. und Ep. unselbständig (s. Bardoney)

B ardoney (-)

$\begin{array}{ll}1952 & \text { nur noch Reste } \\ 1931 & \text { c. } 2640 \text { (Rest) } \\ 1920 & ? ? \\ 1860 & \text { c. } 2360 \\ 1820 & ? \\ 17 / 18 . ~ J h . & ? \\ \text { Va. } & \text { c. } 2240(2 \mathrm{x}) \\ \text { Ep. } & \text { F (oder unselbständig, s. Valeille) }\end{array}$

HD

VD

$\begin{array}{cc}200 \mathrm{~m} & 70 \mathrm{~m} \\ ? & ? \\ ? & ? \\ ? & ? \\ ? & ? \\ 3,3 \mathrm{~km} & 460 \mathrm{~m} \\ 5,5 \mathrm{~km} & 850 \mathrm{~m}\end{array}$




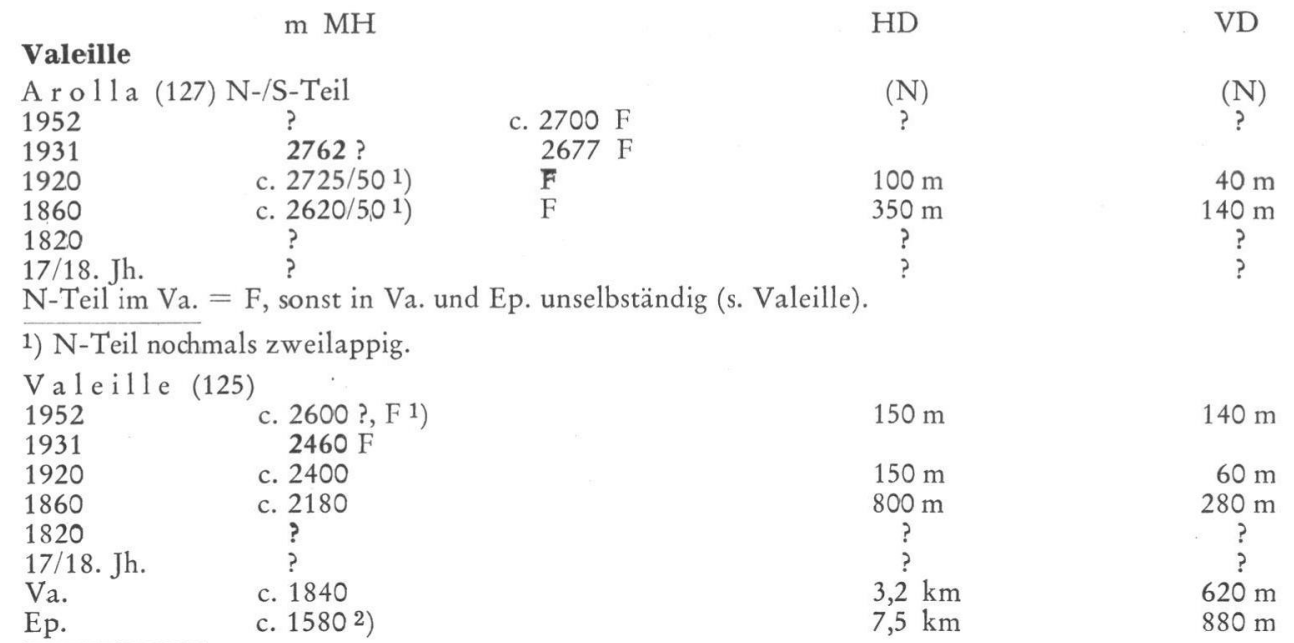

1) $1937=2490$ (Jber. in Boll. Com. glac. 1938).

2) W-Ende des Zungenbeckens von Lillaz bei Champlong.

\section{Valnontey}

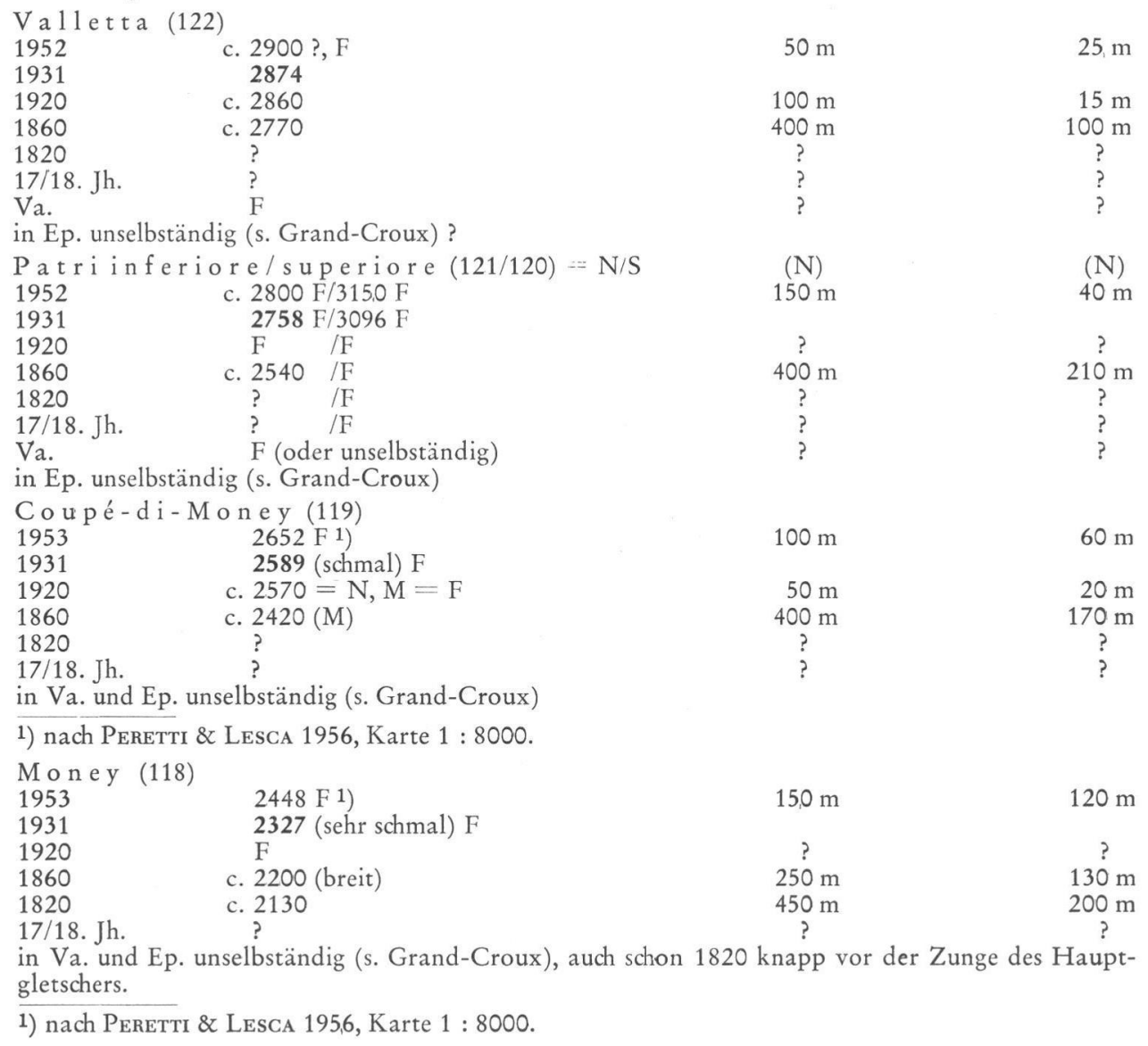


$\mathrm{m} \mathrm{MH}$

Grand-Croux (117)

1953

1931

1920

1860

$\left.1820^{3}\right)$

17/18. Jh.

$\mathrm{Va}$.

Ep.
2244

c. 2220

c. $1970^{2}$ )

c. 1840

?

c. $1670^{4}$ )

c. 14005 )
HD

VD

1) nach Peretti \& Lesca 1956, Karte $1: 8000$.

2) im Gelände markiert durch Block nahe Zungenende, Maximum 1866; vgl. auch Abb. 2.

3) nach alten Quellen (vgl. z. B. SACCO 1921) wurde das historische Maximum 1817/18 erreicht.

4) im Ortsteil Valnontey, hier namengebend für dieses Stadium.

5) im Ortsteil Epinel, hier namengebend für dieses Stadium.

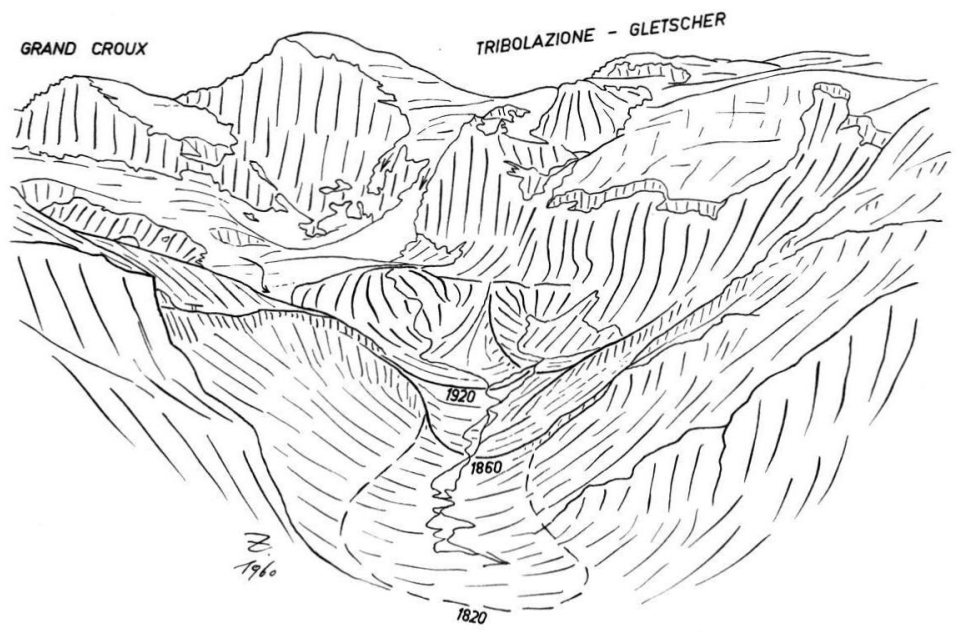

Abb. 2. Oberstes Valnontey von NO (Aufstieg zur A. Money); Gran-Croux- und Tribolazione-Gletscher. m $\mathrm{MH}$

HD

Tribolazione (116)

1953

1931

1920

2598 F 1)

2304 (z. T. regeneriert)

c. 2280

1860 bis Ep. unselbständig (s. Grand-Croux).

$\begin{array}{cc}150 \mathrm{~m} & 50 \mathrm{~m} \\ 80 \mathrm{~m} & 25 \mathrm{~m} \\ 900 \mathrm{~m} & 270 \mathrm{~m} \\ 1,9 \mathrm{~km} & 400 \mathrm{~m} \\ ? & ? \\ 6,5 \mathrm{~km} & 570 \mathrm{~m} \\ 12,5 \mathrm{~km} & 840 \mathrm{~m}\end{array}$

1) nach Peretti \& Lesca 1956, Karte $1: 8000.1960$ gab es u n t e r h a 1 b der Felswand wieder ein Stück regenerierten Gletscher (min. c.2350, vgl. Abb. 2), die Verhältnisse hier scheinen also sehr zu wechseln.

L a u s o n (112) 1)

$\begin{array}{ll}1952 & \text { c. } 3025 \text { ? } \\ 1931 & \text { c. } 29002 \text { ) } \\ 1920 & \text { c. } 2880 \\ 1860 & \text { c. } 2790 \text { (rechts doppelt) } \\ 1820 & \text { c. } 2775 \\ 17 / 18 . ~ J h . & ? \\ \text { Va. } & \text { c. } 25603 \text { ) }\end{array}$

in Ep. wahrscheinlich unselbständig (s. Grand-Croux) oder F.

$\begin{array}{cc}400 \mathrm{~m} & 125 \mathrm{~m} \\ & \\ 200 \mathrm{~m} & 20 \mathrm{~m} \\ 500 \mathrm{~m} & 110 \mathrm{~m} \\ 550 \mathrm{~m} & 125 \mathrm{~m} \\ ? & ? ? \\ 1,5 \mathrm{~km} & 340 \mathrm{~m}\end{array}$

\footnotetext{
1) bei VANNi etc. 1953 und auf den Karten vor $1931=$ „Gran-Sertz“.

2) $1934=2938$ (Peretti 1935 a).

3) unterhalb vom Rif. V. Sella, dadurch Aufstau des glazifluvialen Schuttes oberhalb davon.
} 
Grand-Eyvia-Tal

m $\mathrm{MH}$

HD

VD

Tráio (107)

1952

c. 2500 ?, $\mathrm{T}$

$300 \mathrm{~m}$

$200 \mathrm{~m}$

$110 \mathrm{~m}$

c. 1940

c. 2470 (Moräne)

$80 \mathrm{~m}$

1920

1860

c. 2350 (breit)

$80 \mathrm{~m}$

$40 \mathrm{~m}$

1820

c. 2320 (dreizipflig)

$200 \mathrm{~m}$

$70 \mathrm{~m}$

17/18. Jh.

c. 2170 (dreizipflig) 1)

$550 \mathrm{~m}$

c. 1830

?

$220 \mathrm{~m}$

$\mathrm{Va}$

$1,5 \mathrm{~km}$

in Ep. unselbständig (dicht neben Grand-Croux) oder F wenig oberhalb.

1) dieser Stand vielleicht älter, Formen des N-Zipfels innen sehr frisch, wie sonst nur 1860 (bei der Steilheit des Geländes überhaupt manches anders als sonst üblich), darüber gibt es aber noch eine sehr deutliche Moräne in diesem Zipfel weit unterhalb 1920. BescheL 1958 verzeichnet bei diesem Gletscher die Moränen z. T. ganz anders, als man sie im Gelände vorfindet.

Grivolet t a (106)

1952 c. 3000

1931 c. 29501

1920 c. 2910?

$100 \mathrm{~m}$

$50 \mathrm{~m}$

1860

c. 2780

$100 \mathrm{~m}$

$40 \mathrm{~m}$

1820

?

$17 / 18$. Jh

$350 \mathrm{~m}$

$170 \mathrm{~m}$

$\mathrm{Va}$.

c. 2600 ?

?

$900 \mathrm{~m}$

$350 \mathrm{~m}$

in Ep. schmal und direkt neben Tráio, Ende = ?

1) $1934=2970($ Peretti $1935 \mathrm{a})$.

\section{Valsavaranche}

Timorion (103)

1951

1931

1920

1860

$3071^{1)} \mathrm{Fp}$

$2987 \mathrm{Fp}$

c. 2850

2850

$200 \mathrm{~m}$

$80 \mathrm{~m}$

1820

c. 2820

$600 \mathrm{~m}$

?

$17 / 18$. Jh.

?

$650 \mathrm{~m}$

c. $2700^{2}$ )

$1,1 \mathrm{~km}$

$170 \mathrm{~m}$

?

$290 \mathrm{~m}$

in Ep. wahrscheinlich unselbständig (s. Grand-Etrét) oder F unterhalb A. Leviona inferiore.

1) 1951 hier und beim nächsten Gletscher nach MoRETTI 1952.

2) abgeklemmt durch die beiden Nachbargletscher (Inferno und Grand-Neiron).

Grand-Neiron (102) ${ }^{1}$ )

1951

2820

$100 \mathrm{~m}$

$20 \mathrm{~m}$

1931

2802

1920 c. 2770

$150 \mathrm{~m}$

$30 \mathrm{~m}$

1860 c. 2700 (B)

$600 \mathrm{~m}$

$100 \mathrm{~m}$

1820 c. 2690 (B)

1770 c. 2685 (B)

1680 c. 2685 (B)

1600 c. 2680 (B)

$\mathrm{Va}$.

c. 23702 )

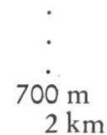

$110 \mathrm{~m}$

$115, \mathrm{~m}$

$115, \mathrm{~m}$

$120 \mathrm{~m}$

in Ep. wie Timorion.

1) vgl. Abb. 3 und Beschel 1958, Fig. 4.

2) knapp oberhalb der A. Leviona di mezzo, zusammen mit den Gletschern vom Col Lauson (damals $2 \mathrm{~km}$ ) und aus dem V. dell'Inferno (dieser dann 2,5 km lang).

Montandey né (101) ${ }^{1}$ )

N-Teil:

$\left.1950 \quad 3070^{2}\right) \mathrm{Fp}$

$150 \mathrm{~m}$

$100 \mathrm{~m}$

1931

$2974 \mathrm{~F}$

1) vgl. Beschel 1958, Fig. 2.

2) 1950 hier und bei den folgenden Gletschern nach Jber. in Boll. Com. glac. 1952. 


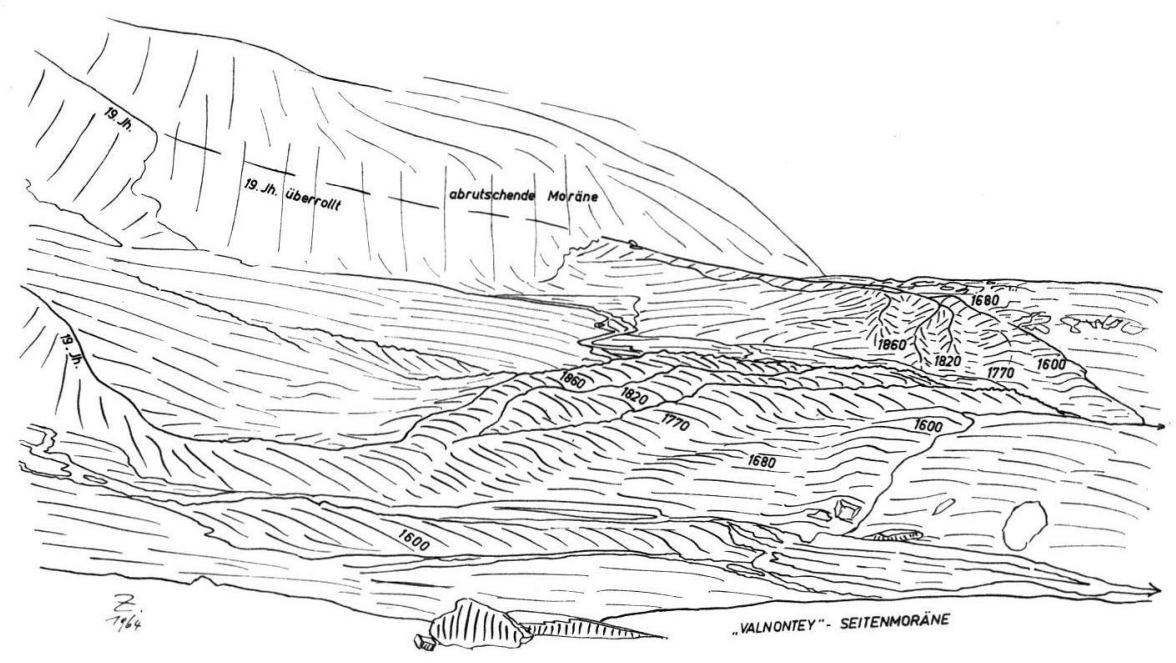

Abb. 3. Die Moränen des Gran-Neiron-Gletschers von N; Blick von der „Epinel“- über die „Valnontey“-Seitenmoräne auf die historischen Moränen (Datierung der historischen Moränen nach BESCHEL 1958).

\begin{tabular}{|c|c|c|c|}
\hline & $\mathrm{m} \mathrm{MH}$ & HD & VD \\
\hline 1920 & $\mathrm{~F}$ & ? & $?$ \\
\hline 1860 & c. 2850 & $400 \mathrm{~m}$ & $120 \mathrm{~m}$ \\
\hline 1820 & $?$ & $?$ & ? \\
\hline 1770 & c. 2790 (vgl. S) & $500 \mathrm{~m}$ & $180 \mathrm{~m}$ \\
\hline 17. Jh. & ? & ? & ? \\
\hline $\begin{array}{l}\text { Va. } \\
\text { S-Teil: }\end{array}$ & c. 2520 (breit) & $1,5 \mathrm{~km}$ & $450 \mathrm{~m}$ \\
\hline 1952 & $\mathrm{~F}$ & ? & ? \\
\hline 1931 & $2895 \mathrm{~F}$ & & \\
\hline 1920 & $\mathrm{~F}$ & ? & ? \\
\hline 1860 & c. $2780(\mathrm{~B}=2740)$ & $450 \mathrm{~m}$ & $115 \mathrm{~m}$ \\
\hline 1820 & ? (B) & ? & ? \\
\hline 1770 & c. 2730 (B) & $600 \mathrm{~m}$ & $165, \mathrm{~m}$ \\
\hline 17. Jh. & c. $2720(\mathrm{~B})$ & $650 \mathrm{~m}$ & $175 \mathrm{~m}$ \\
\hline Va. & c. $2450(\mathrm{~B}=$,Larstig" $)$ & $1,7 \mathrm{~km}$ & $450 \mathrm{~m}$ \\
\hline in Ep. 1 & unselbständig (s. Grand-Etrét). & & \\
\hline Lave & D) 1) & & \\
\hline $\begin{array}{l}1952 \\
1931\end{array}$ & $\begin{array}{l}2720 \mathrm{~F} \\
\text { c. } 2620 \mathrm{~F} 2)\end{array}$ & $300 \mathrm{~m}$ & $100 \mathrm{~m}$ \\
\hline 1920 & c. 26003 ) & $80 \mathrm{~m}$ & $20 \mathrm{~m}$ \\
\hline 1860 & c. 2525 & $450 \mathrm{~m}$ & $95 \mathrm{~m}$ \\
\hline 1820 & c. 2500$\} \quad(B=\# 1850 ")$ & $500 \mathrm{~m}$ & $120 \mathrm{~m}$ \\
\hline 1770 & c. 2480 (B) & $530 \mathrm{~m}$ & $140 \mathrm{~m}$ \\
\hline 1680 & c. 2480 (B) & $550 \mathrm{~m}$ & $140 \mathrm{~m}$ \\
\hline 1600 & c. 2470 (B) & $580 \mathrm{~m}$ & $150 \mathrm{~m}$ \\
\hline Va. & c. 2300 (breit) $\left.{ }^{4}\right)$ & $1 \mathrm{~km}$ & $320 \mathrm{~m}$ \\
\hline
\end{tabular}

in Ep. unselbständig (s. Grand-Etrét).

1) vgl. BESCHEL 1958, Fig. 2.

2) nach den amtlichen topogr. Karten 1:25000 wäre 1931 = 2507, Photos von 1926 und 1928 (Bossolasco 1929) zeigen den langen, schmalen Zipfel von 1931 aber nicht; da man sich sonst auf die amtlichen Karten wirklich verlassen kann, scheint 1931 entweder ein Firnrest oder ein Rest von Eislawinen weiter hinunter gereicht zu haben.

3) diese Moräne ist auf den genannten Photos gut zu erkennen, aber heute nicht mehr erhalten; vielleicht durch Eislawine zerstört ?

4) BESCHEL nennt Va.-Seitenmoräne bei $2340 \mathrm{~m} \mathrm{MH}$ „Larstig“. 
m MH

Gra n-P a r a d is o (99)

$\begin{array}{lc}1950 & \text { c. } 2960 \text { ?, F } \\ 1931 & 2896 \\ 1920 & \text { c. } 2880 \\ 1860 & \text { c. } 2800 \\ 1820 & \text { c. } 2780 \\ 17 / 18 . \text { Jh. } & \text { ? } \\ \text { Va. } & \text { c. } 2575 \text { (B = „Larstig ") } \\ \text { in Ep. unselbständig (s. Grand-Etrét) }\end{array}$

Mon tcorvé (98) N-Teil 1)

1952

1931

1920

1860

1820

1770

1680

vor 1600 ?

$\mathrm{Va}$.

in Ep. unselbständig (s. Grand-Etrét).

1) vgl. Beschel 1958, Fig. 1.

2) $1950=2800$.

Moncia i $(97)$

$\begin{array}{lc}1952 & \left.\text { c. } 2800^{1}\right) \\ 1931 & 2734 \\ 1920 & \text { c. } 2730 \\ 1860 & \text { F } \\ 1820 & \text { F } \\ 17 / 18 . \text { Jh. } & \text { F }\end{array}$

in Va. und Ep. unselbständig (s. Grand-Etrét).
HD

VD

$150 \mathrm{~m}$

$50 \mathrm{~m}$

$400 \mathrm{~m}$

$450 \mathrm{~m}$

?

$1,3 \mathrm{~km}$

$200 \mathrm{~m}$

$200 \mathrm{~m}$

$550 \mathrm{~m}$

?

$750 \mathrm{~m}$

? $900 \mathrm{~m}$

?
$60 \mathrm{~m}$

$15 \mathrm{~m}$

$100 \mathrm{~m}$

$120 \mathrm{~m}$

?

$320 \mathrm{~m}$

$50 \mathrm{~m}$

$45 \mathrm{~m}$ $130 \mathrm{~m}$

?

$170 \mathrm{~m}$

?

$230 \mathrm{~m}$

?

1) $1950=2785$

B reuil-occid. (96)

1952

1931

?

1920

2692

1860

c. 2670

c. 2640

1820

?

17/18. Jh.

?

$250 \mathrm{~m}$

$65 \mathrm{~m}$

$60 \mathrm{~m}$

?

$?$

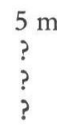

in Va. und Ep. unselbständig (s. Grand-Etrét).

Grand-Etrét (95) 1)

$1950 \quad 2432$

19312420

1920 c. $2380(\mathrm{~B}=$ c. 2400$)$

1860 c. 2300 (B)

1820 ? (B)

1770 c. 2290 (B)

17. Jh. ? (B)

$\begin{array}{ll}\mathrm{Va} . & \text { c. } 2070\end{array}$

Ep. c. $1530-1580^{2}$ )

$30 \mathrm{~m}$

$10 \mathrm{~m}$

$80 \mathrm{~m}$

$250 \mathrm{~m}$

?

$50 \mathrm{~m}$

?

1) vgl. Abb. 4.

2) Degioz-Tignet, mehrere Wälle.

\section{Val di Rhêmes}

$\begin{array}{ll}\text { L a v a s e y } & (86) \\ 1951 & 26202) \\ 1931 & \text { c. } 2575 \\ 1920 & \text { c. } 2570\end{array}$

$200 \mathrm{~m}$

$45 \mathrm{~m}$

1) vgl. Abb. 5, linke Seite.

2) nach Jber. in Boll. Com. glac. 1952, nur sehr schmaler Zipfel.

$60 \mathrm{~m}$

$5 \mathrm{~m}$ 


$\begin{array}{ll} & \text { m MH } \\ 1860 & \text { c. } 2460 \\ 1820 & \text { c. } 2460 \\ 1770 & ? \\ 17 . \text { Jh. } & \text { c. } 2430 \text { ? } 3 \text { ) } \\ \text { Va. } & \text { F } \\ \text { Ep. } & \text { c. } 1400-1480 \text { 4) }\end{array}$

$\begin{array}{cc}\mathrm{HD} & \mathrm{VD} \\ 750 \mathrm{~m} & 115 \mathrm{~m} \\ 800 \mathrm{~m} & 115 \mathrm{~m} \\ ? & ? \\ 1 \mathrm{~km} & 145 \mathrm{~m} \\ ? & ? \\ 16-17 \mathrm{~km} & 1100-1175 \mathrm{~m}\end{array}$

3) stark begrünt, Vegetation von derjenigen der Umgebung nicht mehr zu unterscheiden, Mittelmoräne zum W-Teil des Fond-Gletschers deutlich, Endmoräne nur an einzelnen Buckeln einigermaßen zu erkennen. Damals scheint es hier nur zwei Gletscher gegeben zu haben, im $\mathrm{O}$ den Lavassey- (einschließlich Fond-O), im W den über die Felskante herunterreichenden TsanteléinaGletscher (einschließlich Fond-W); dazwischen liegt die einzige Mittelmoräne.

4) Proussaz-Crêton, zwei jeweils nur auf einer Seite des Baches neben schluchtartigem Talgrund entwickelte deutliche Wälle.

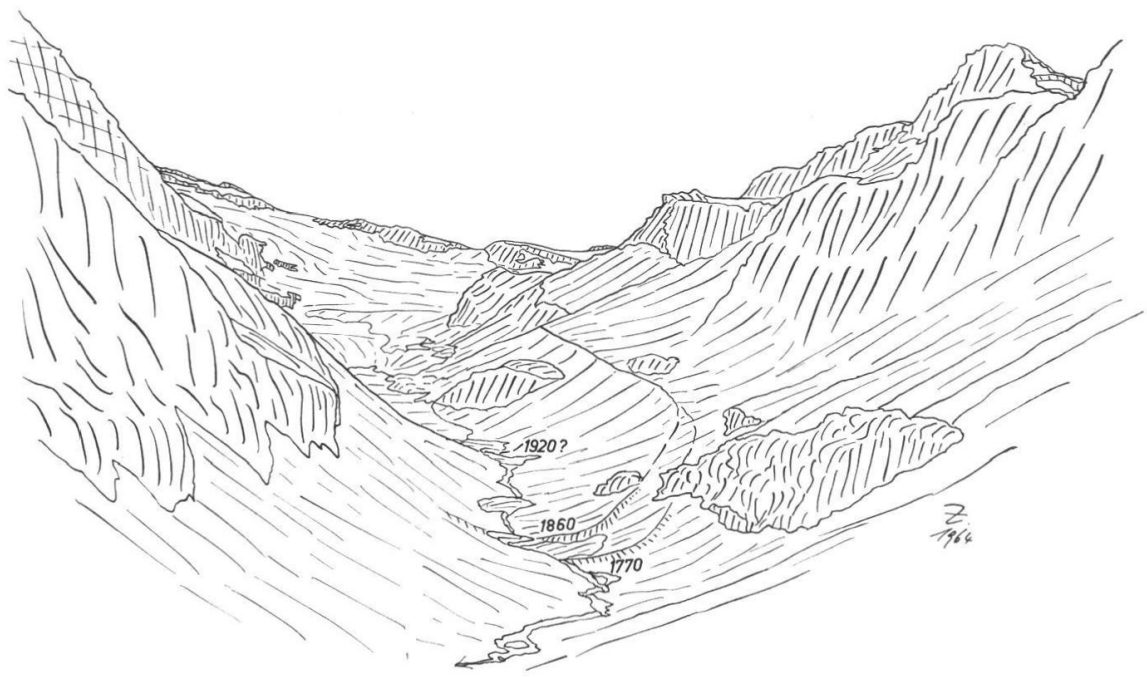

Abb. 4. Oberstes Valsavaranche von N, Moränen des Grand-Etrét-Gletschers

(Datierung nach BESCHEL 1958).

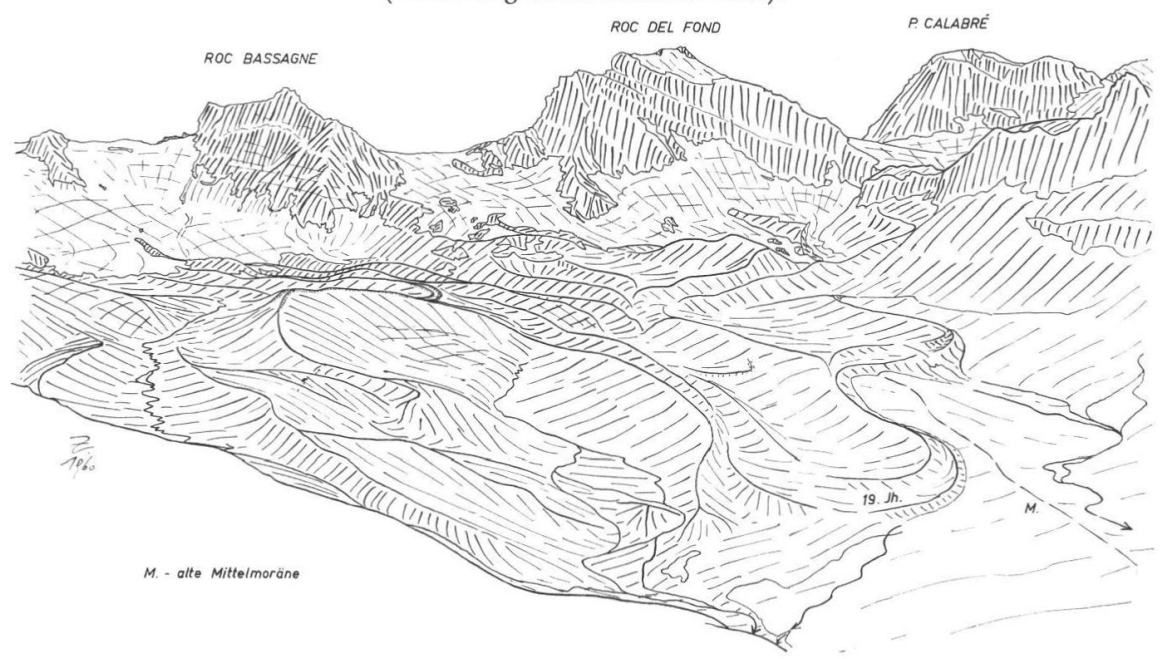

Abb. 5. Oberstes Val di Rhêmes von NO. Der Doppelmoränenbogen links im Mittelgrund ist die 1920-Moräne des Ostteiles vom Fond-Gletscher. 
m $\mathrm{MH}$

$\mathrm{HD}$

VD

F on d $=$ Rhêmes (84/85) O-Teil 1)

$\begin{array}{ll}1952 & ? \\ 1931 & \text { c. } 2650 \text { (schmal) } \\ 1920 & \text { c. } 2640 \text { (doppelt) } \\ 1860 & \text { c. } 2470 \text { (schmal) } \\ 1820 & \text { c. } 2460 \\ 1770 & ? \\ 17 . \text { Jh., in Va. und Ep. unselbständig (s. Lavassey). }\end{array}$

$\begin{array}{cr}? & ? \\ 150 \mathrm{~m} & 10 \mathrm{~m} \\ 1,1 \mathrm{~km} & 180 \mathrm{~m} \\ 1,15 \mathrm{~km} & 190 \mathrm{~m} \\ ? & ?\end{array}$

1) vgl. Abb. 5 .

Ts a n teléina (83)

1952

1930

2745 ?

1920

c. 2700 (Moränen)

1860

c. $\left.2570(2590)^{1}\right)$

c. 2490 (breit)

c. 2490

1820

17. Jh.

?

c. $2480^{2}$ )

$100 \mathrm{~m}$

$45 \mathrm{~m}$

$150(400) \mathrm{m}$

$500 \mathrm{~m}$

$550 \mathrm{~m}$

?

$600 \mathrm{~m}$

$130(110) \mathrm{m}$ $210 \mathrm{~m}$ $210 \mathrm{~m}$ ? $220 \mathrm{~m}$

in Va. und Ep. unselbständig (s. Lavassey).

1) in zwei verschieden breiten und verschieden langen Zipfeln; der schmale und viel längere nördliche (...) stark gebogen; beide enden unterhalb hoher Felsstufe.

2) stark begrünt, Vegetation von derjenigen der Umgebung fast nicht mehr zu unterscheiden, seitliche Teile der Endmoräne und Seitenmoränen (vor allem auf der N-Seite) deutlich.

Golet t a (82)

$\begin{array}{ll}1951 & \left.2714(=\text { See })^{1}\right) \\ 1930 & \text { c. } 2700 \\ 1920 & ? \\ 1860 & \text { c. } 2630(?) \\ 1820 & \text { c. } 2560 \\ 17 / 18 . \text { Jh. } & \text { ? } \\ \text { Va. } & \text { ? (F ?) } \\ \text { in Ep. unselbständig (s. Lavassey). }\end{array}$

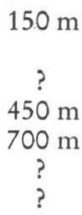

$15 \mathrm{~m}$

1) nach Jber. in Boll. Com. glac. 1952.

\section{Valgrisanche}

B a s s a $(65)$

$\begin{array}{ll}1952 & \text { c. } 2780 \\ 1930 & \text { c. } 2750 \\ 1920 & \text { c. } 2690 \text { (vierzipflig) } \\ 1860 & \text { c. } 2630 \text { (dreizipflig) } \\ 1820 & ? \\ 17 / 18 . \text { Jh. } & ?\end{array}$

$50 \mathrm{~m}$

$30 \mathrm{~m}$

in Va. und Ep. unselbständig (s. Vaudet).

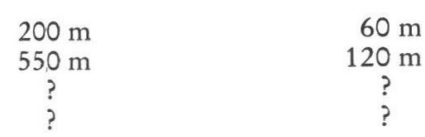

$\mathrm{V}$ a ud e t (63) 1)

$\begin{array}{ll}1952 & \text { c. } 2340 \mathrm{~T} \\ 1930 & 2306 \mathrm{~T} \\ 1920 & \mathrm{~T} \\ 1860 & \mathrm{~T} ? \\ 1820 & \text { c. } 2270 \\ 1770 & ? \\ 17 . \text { Jh. ? } & \text { c. } 2240 \\ \text { Va. } & \text { c. } 1890 \text { ? 2) } \\ \text { Ep. } & \text { c. } 1570-16003)\end{array}$

$250 \mathrm{~m}$

$35 \mathrm{~m}$

1) vgl. Abb. 6 .

2) bei le Chalet kleines Zungenbecken vorhanden, Moränen meist durch mächtige Hangschuttfächer verdeckt.

3) Ceré und la Betthaz-Planté. 


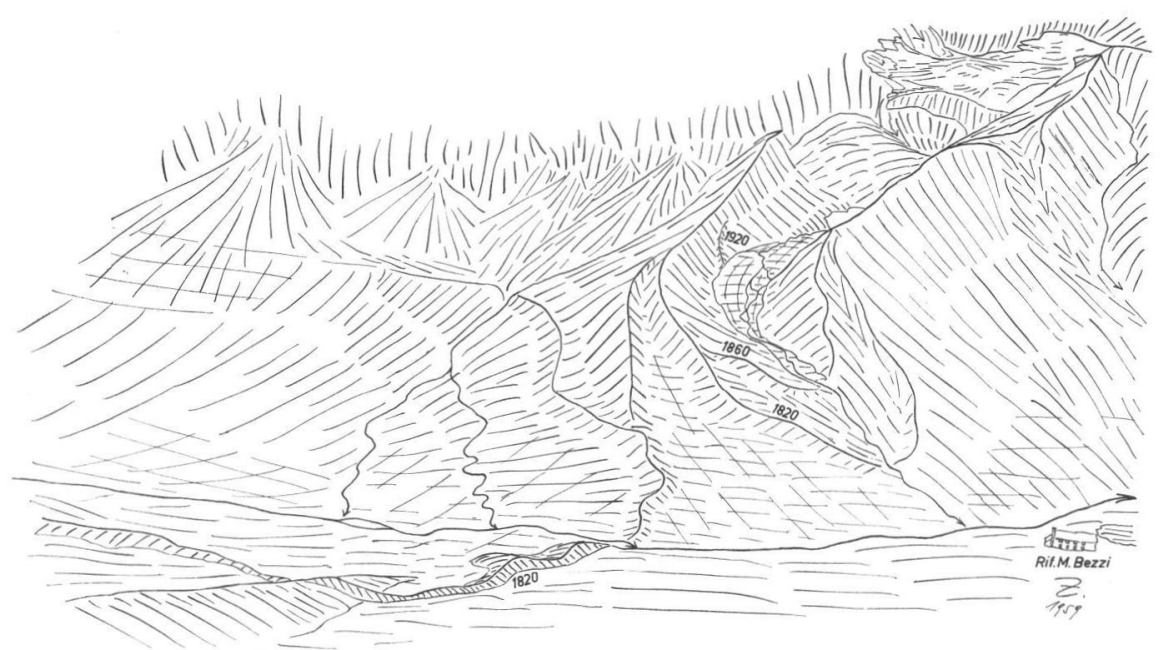

Abb. 6. Oberstes Valgrisanche von O, links die Moränen des Vaudet-Gletschers (heutiges Toteis links außerhalb), rechts diejenigen des Plattes-de-Chamois-Gletschers.

$\mathrm{m}$ MH

Plat tes-de-Chamois (59) ${ }^{1}$ )

$\begin{array}{ll}1952 & \text { c. } 2400 \text { ? } \\ 1930 & \text { c. } 2370 \text { (schmal) } \\ 1920 & \text { c. } 2330 \\ 1860 & \text { c. } 2300 \\ 1820 & \text { c. } 2270^{2} \text { ) } \\ 1770 & \text { ? }\end{array}$

17. Jh., in Va. und Ep. unselbständig (s. Vaudet).

1) vgl. Abb. 6 .

2) knapp neben Vaudet, diesem z. T. den Weg versperrend.

\section{Rutor-Tal}

$\begin{array}{ll}\text { Rutor (46) }{ }^{1)} & \\ 1952 & 2523 \mathrm{~F} \\ 1929 & 2387 \text { (See) } \\ 1920 & 2387 \text { (Moräne im See) } \\ 1860(2 \mathrm{x}) & \text { c. } 2140 \text { (schmal) } \\ & \text { c. } 2140 \text { (mittel) } \\ 1820 & \text { c. } 2130 \text { (breit) } \\ 17 / 18 . \text { Jh. } & ? \\ \text { Va. } & \text { c. } 1600 \text { (?) } 2 \text { ) } \\ \text { Ep. } & \text { c. } 1450 \text { (Thuile-Entrèves) }\end{array}$

HD

VD

$100 \mathrm{~m}$

$30 \mathrm{~m}$

$60 \mathrm{~m}$

$200 \mathrm{~m}$

$350 \mathrm{~m}$

?
$40 \mathrm{~m}$

$70 \mathrm{~m}$ $100 \mathrm{~m}$

?

1) vgl. Karten in SACCo 1934 a und Baretti 1880 a; dazu Abb. 7.

2) unterhalb la Joux, seitliche Teile der Endmoräne erhalten, unteres Ende F.

Val de la Lex Blanche - Val Veni (vgl. Abb. 8-10)

Estellet te (30)

1952 ?

1929 c. 2450

1920 c. 2420

1860 c. 2370

1820 c. 2340

17/18. Jh. c. 2245

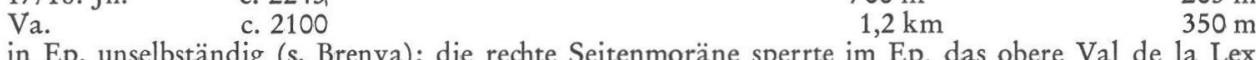

$200 \mathrm{~m} \quad 135 \mathrm{~m}$

$150 \mathrm{~m} \quad 0 \mathrm{~m}$

$1,2 \mathrm{~km} \quad 250 \mathrm{~m}$

$1,2 \mathrm{~km} \quad 250 \mathrm{~m}$

$1,25 \mathrm{~km} \quad 260 \mathrm{~m}$

? ?

$3,5 \mathrm{~km} \quad 790 \mathrm{~m}$

$7 \mathrm{~km} \quad 940 \mathrm{~m}$ Blanche nach unten $a b$. 


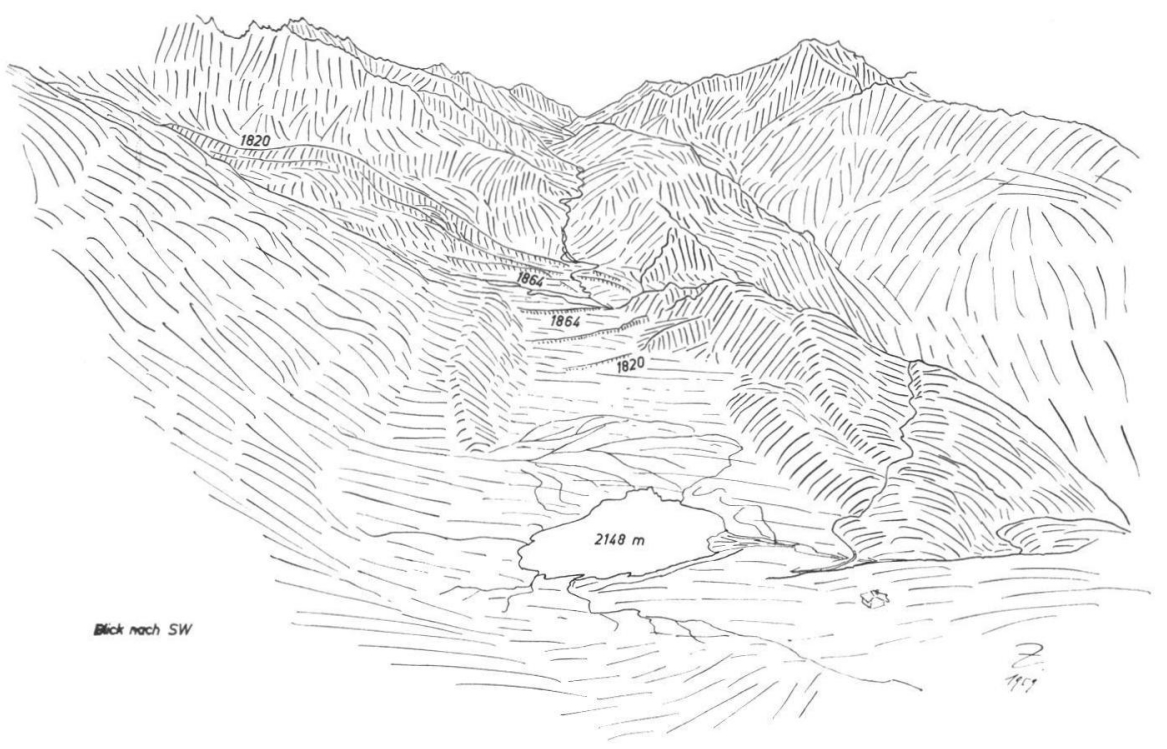

Abb. 7. Rutor-Gebiet; weit links außerhalb der heutige Gletscher, unterhalb der im Bilde linken Felsstufe die Moränen aus dem 19. Jh., das mächtige Stück der Moräne von 1820 wohl zum großen Teil älter.

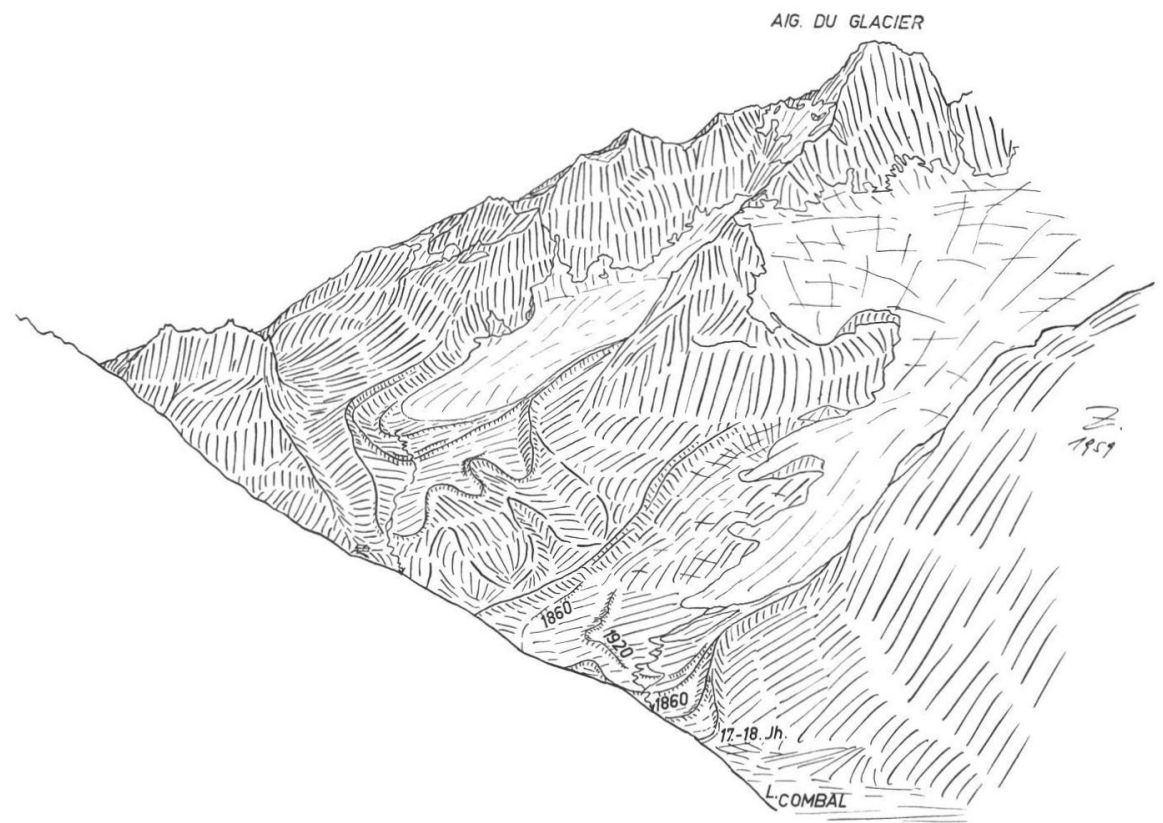

Abb. 8. Val de la Lex Blanche (=Oberteil des Val Veni) von O. Moränen des Estellette- und des Lex-Blanche-Gletschers. Die Moränen des Estellette-Gletschers (von innen nach außen) gehören zu 1920, 1860 (kurz), 1820, 17.-18. Jh. (dreizipflig, z. T. doppelt) und Valnontey. Die unbeschriftete Moräne des Lex-Blanche-Gletschers vorn rechts ist von 1820. 


\section{$\mathrm{m} \mathrm{MH}$}

L e x - B la n che (29)

$\begin{array}{ll}1951 & 2080 \text { 1) } \mathrm{T} \\ 1929 & 2031 \\ 1920 & \text { c. } 2005 \\ 1860 & \text { c. } 1990 \\ 1820 & \text { c. } 1975 \\ 17 / 18 . \mathrm{Jh} . & \text { c. } 1965 \\ \text { Va. } & \text { c. } 1960 \text { ? 2) }\end{array}$

HD

VD

$300 \mathrm{~m}$

$50 \mathrm{~m}$

$150 \mathrm{~m}$

$25, \mathrm{~m}$

$250 \mathrm{~m} \quad 40 \mathrm{~m}$

$400 \mathrm{~m} \quad 55 \mathrm{~m}$

$450 \mathrm{~m} \quad 65 \mathrm{~m}$

c. $1,5 \mathrm{~km} \quad 70 \mathrm{~m}$

in Ep. unselbständig (s. Brenva).

1) nach Jber. in Boll. Com. glac. 1952.

2) versunken im "Lac Combal“ genannten Aufschüttungsgebiet oberhalb der das Tal sperrenden Miage-Seitenmoräne.

Miage (23) 1 )

N-Teil:

1952

1929

?

1920

c. 1780

1860

c. 1775 (?)

1820

c. 1765

17/18. Jh.

c. 1730

c. 1700 ? 2 )

Va. und Ep. s. S-Teil.

S-Teil:

1952

?

1929

c. 1740

1920 c. 1730

1860

1820

c. $1675 / 1710^{3}$ )

17/18. Jh.

c. $1620 / 1680$

$\mathrm{Va}$.

c. 15,80

c. 14904 )

in Ep. unselbständig (s. Brenva).

$\begin{array}{rr}? & ? \\ 20 \mathrm{~m} & 5 \mathrm{~m} \\ 80 \mathrm{~m} & 15 \mathrm{~m} \\ 200 \mathrm{~m} & 50 \mathrm{~m} \\ 350 \mathrm{~m} & 80 \mathrm{~m}\end{array}$

1) vgl. auch die Karte 1:10000 in Barettr $1880 \mathrm{~b}$.

2) vorderste Teile der Endmoräne und linke Seitenmoräne unter dem Fréiney-Schuttfächer versunken, Höhenwert interpoliert.

3) S-Teil zeitweilig nochmals zweizipflig, deshalb hier zwei Werte.

4) Reste zweier flacher Wälle bei den Cas. Purtud, im Staugebiet oberhalb der Brenva-Moränen, erkennbar (vgl. Abb. 9); die südseitige Eisrandterrasse ist im ganzen Verlauf deutlich.

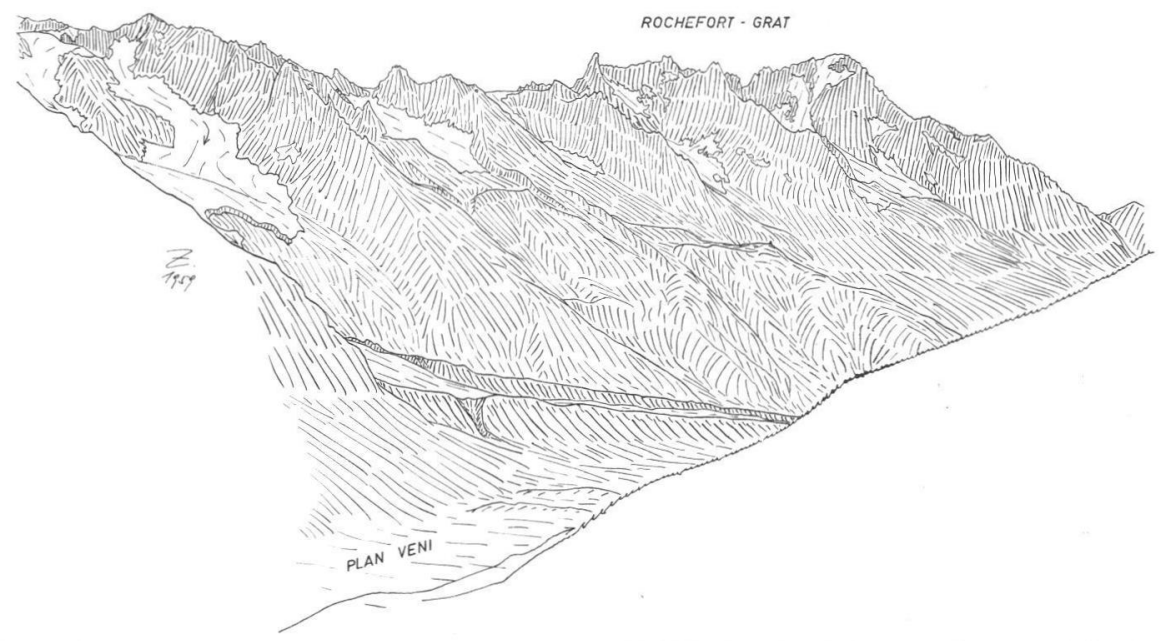

Abb. 9. Blick vom Standort der Abb. 8 nach NO ins Val Veni und auf den mittleren Teil der MontBlanc-Gruppe. Im Talgrund die „Valnontey“-Moränen des Miage-Gletschers, dahinter die rezenten Moränen des Brenva-Gletschers. 


\begin{tabular}{|c|c|c|c|}
\hline \multicolumn{2}{|r|}{$\mathrm{m} \mathrm{MH}$} & $\mathrm{HD}$ & VD \\
\hline $\begin{array}{l}1952 \\
1940\end{array}$ & $\begin{array}{l}? \\
1355\end{array}$ & $\stackrel{?}{?}-100 \mathrm{~m}$ & $\stackrel{?}{?} \mathrm{~m}$ \\
\hline 1929 & 1379 & & \\
\hline 1920 & $14202)$ & $-400 \mathrm{~m}$ & $-40 \mathrm{~m}$ \\
\hline 1860 & c. 13453$)$ & $250 \mathrm{~m}$ & $35 \mathrm{~m}$ \\
\hline 1820 & c. 1340 3) & $300 \mathrm{~m}$ & $40 \mathrm{~m}$ \\
\hline 17/18. Jh. & c. 1340 3) 4) & $300 \mathrm{~m}$ & $40 \mathrm{~m}$ \\
\hline Va. & c. 1190 (Villette) 5) & $3,2 \mathrm{~km}$ & $190 \mathrm{~m}$ \\
\hline Ep. & c. 1100 (Verrand-S' $\left.{ }^{6}\right)$ & $6 \mathrm{~km}$ & $280 \mathrm{~m}$ \\
\hline
\end{tabular}

1) nach CAPELLo 1941 stieß der Gletscher von 1920 bis 1940 etwa $500 \mathrm{~m}$ vor (HD und VD deshalb gegenüber sonst negativ); bis 1960 ging er wieder ähnlich weit zurück; über den Bergsturz, der den außergewöhnlichen Vorstoß 1920-1940 verursachte, siehe am kürzesten SACCO 1942, S. 6 ff.; aus der Zeit kurz nach dem Bergsturz stammt auch der kleine, seitliche Ausbruch ganz im W der das Val Veni sperrenden rechten Seitenmoräne (vgl. Abb. 9).

2) nach Capello 1941 und Valbusa 1927.

3) 1860 (eigentlich 1846 und 1853), 1820 (eigentlich 1818) und 17./18. Jh. nach Marengo 1881 und SILVESTRI 1925. (HD) und der Karte $1: 25000$ des IGM (MH und VD).

4) im 16. Jh. stieß der Gletscher erstmals wieder in die Talsohle vor, zerstörte einen Weiler und die umliegenden Kulturen (im 14. und 15. Jh. war die Talsohle "coltivato"; vgl. SACCo 1919 und 1942).

5) nur westlich vom Fluß erhalten, vgl. Abb. 10 (dort „V.“).

6) gleichzeitig wurde das Val Ferret nach unten abgesperrt (Aufschüttungs-Talboden).

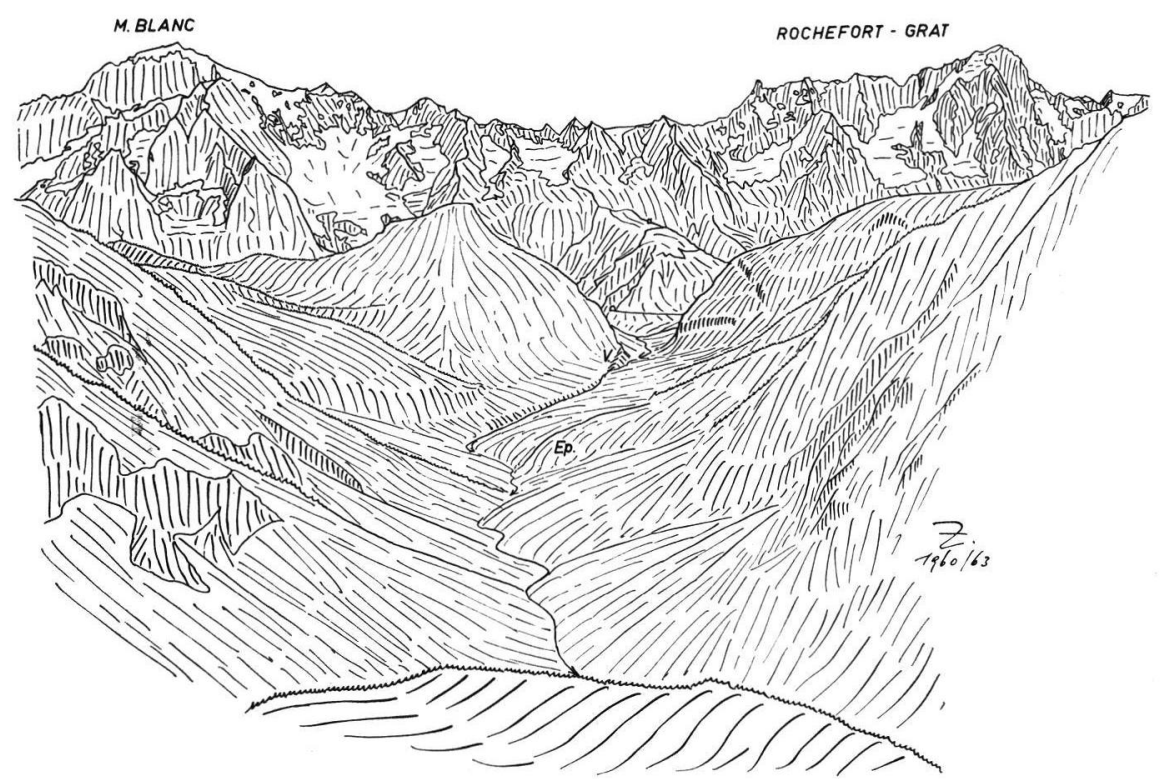

Abb. 10. Blick von der Testa d'Arpy (=von SSO) auf das oberste Valdigna um Courmayeur und auf die Mont-Blanc-Gruppe. V = „Valnontey“-Moräne von Courmayeur-Villette, Ep. = „Epinel“Moränen von Courmayeur-Verrand.

Val Ferret (vgl. Abb. 11)

Triolet (4)

1952

1947

c. 2010 T 1 )

$1994 \mathrm{~T}$

c. 1960 (Moräne) ${ }^{1)}$

1929

c. 1925 (Moräne) 1 )

$250 \mathrm{~m}$

$50 \mathrm{~m}$

1926

1) nach Peretti \& LesCa 1953, Karte $1: 5000$.

$150 \mathrm{~m}$

$35 \mathrm{~m}$

$200 \mathrm{~m}$

$35 \mathrm{~m}$ 


\begin{tabular}{llrr}
\hline & & & \\
& $\mathrm{m} \mathrm{MH}$ & $\mathrm{HD}$ & $\mathrm{VD}$ \\
1920 & c. 1880 (schmal) & $350 \mathrm{~m}$ & $80 \mathrm{~m}$ \\
1860 & c. 1820 & $750 \mathrm{~m}$ & $140 \mathrm{~m}$ \\
1820 & c. 1815 & $800 \mathrm{~m}$ & $145 \mathrm{~m}$ \\
1770 & c. 1805, & $950 \mathrm{~m}$ & $155 \mathrm{~m}$ \\
$17 / 18 . \mathrm{Jh}$. & c. $\left.1790^{2}\right)$ & $1050 \mathrm{~m}$ & $170 \mathrm{~m}$ \\
Va. & c. $1740^{3}$ ) & $3,1 \mathrm{~km}$ & $220 \mathrm{~m}$ \\
Ep. & c. 1650 (Frébouzie) & $4,4 \mathrm{~km}$ & $310 \mathrm{~m}$
\end{tabular}

2) im 16. Jh. stieß der Gletscher wieder ins Tal vor und überfuhr dabei eine Alm (SAcco 1942); noch $1717 \mathrm{gab}$ es oberhalb der Triolet-Gletscherzunge einen Stausee (vgl. Anm. 3 beim folgenden Gletscher).

3) Va.-Moräne mit doppelt gewölbter Front, entspricht wohl Triolet und Pré-de-Bar, deshalb trotz gemeinsamen Endes bei beiden Gletschern HD- und VD-Werte aufgeführt.

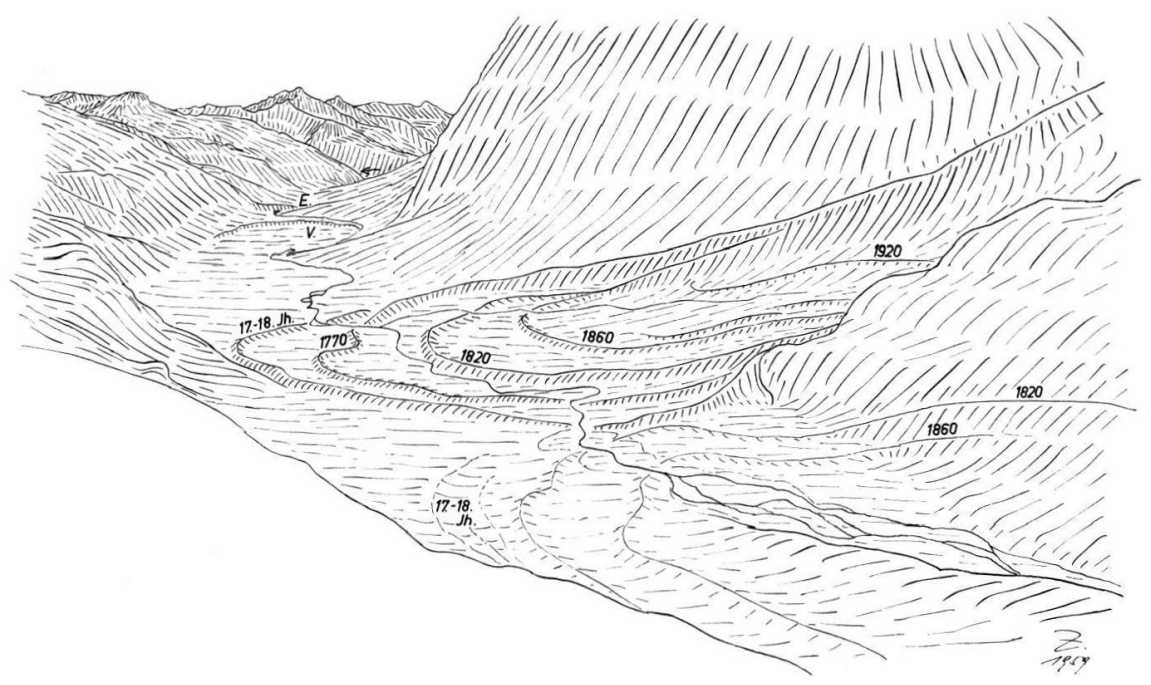

Abb. 11. Val Ferret. Blick vom inzwischen durch eine Lawine zerstörten Rif. Elena nach SW. Im

Vordergrund rechts historische Moränen des Pré-de-Bar-Gletschers, im Mittelgrund diejenigen des Triolet-Gletschers, im Hintergrund die gemeinsamen "Valnontey"- und „Epinel“-Endmoränen dieser beiden Gletscher.

Pré-d e-B a r (3)

\begin{tabular}{|c|c|}
\hline \multirow{2}{*}{\multicolumn{2}{|c|}{$2051^{1)}$}} \\
\hline & \\
\hline 1947 & 2033 \\
\hline 1935 (c.) & c. 1995 (Moräne) 2 ) \\
\hline 1929 & c. 1980 (Moräne) 2 ) \\
\hline 1926 & c. 1970 (Moräne) 2) \\
\hline 1920 & c. 1960 (Moräne) 2) \\
\hline 1860 & c. 1860 \\
\hline 1820 & c. 1850 \\
\hline 1770 & c. $\left.1840^{3}\right)$ \\
\hline 17. Jh. & c. 18403 ) \\
\hline Va. & c. $\left.1740^{4}\right)$ \\
\hline Ep. & c. 1650 \\
\hline
\end{tabular}

$\begin{array}{cr}280 \mathrm{~m} & 70 \mathrm{~m} \\ 180 \mathrm{~m} & 55 \mathrm{~m} \\ 70 \mathrm{~m} & 15 \mathrm{~m} \\ & \\ 80 \mathrm{~m} & 10 \mathrm{~m} \\ 100 \mathrm{~m} & 20 \mathrm{~m} \\ 800 \mathrm{~m} & 120 \mathrm{~m} \\ 85,0 \mathrm{~m} & 130 \mathrm{~m} \\ 900 \mathrm{~m} & 140 \mathrm{~m} \\ 900 \mathrm{~m} & 140 \mathrm{~m} \\ 3,8 \mathrm{~km} & 240 \mathrm{~m} \\ 5,1 \mathrm{~km} & 330 \mathrm{~m}\end{array}$

1) nach Peretti \& LesCa 1953, Karte $1: 2500$.

2) nach 1) und CAPELLO 1940.

3) endnahe Teile der linken Seitenmoränen erhalten (vgl. Abb. 11), die Endmoränen vielleicht nie entwickelt oder verschüttet im ehemaligen Stausee oberhalb der Triolet-Moränen (Ausbruch z. B. in der Nacht zum 12. 9. 1717, vgl. SACCO 1919).

4) siehe Anm. 3 bei Triolet. 
Vergleicht man die HD- und VD-Werte der verschiedenen Gletscher mit den Formen der jeweiligen Gletscherumgebung, so kann man einige allge meine Regeln ableiten.

Ein Tal mit großen Firnmulden und heute eisfreien, aber hochgelegenen Verebnungen im Talanfang und auf beiden Talseiten führt bei einer Senkung der Schneegrenze zu einer starken Vergrößerung des Nährgebietes. Müssen sich die so anfallenden großen Eismengen auch noch in ein nur schmales Tal zwängen, so erfolgt ein besonders kräftiger Vorstoß (Beispiele: Va. im Valnontey und Ep. im Val di Rhêmes und im Valgrisanche).

Ein Tal mit stark einseitiger Entwicklung der hochgelegenen Verflachungen ergibt unter sonst gleichen Bedingungen nur weniger kräftige Vorstöße (Ep. im Rutor-Tal).

Noch kürzer sind die Vorstöße bei besonders tiefer Lage der Talsohle (oberstes Valdigna, s. Brenva) oder dann, wenn sich auch durch eine Erniedrigung der Schneegrenze kein wesentlich größeres Einzugsgebiet ergibt (viele der hier mangels genauerer Höhenwerte für die einzelnen Eisstände nicht aufgeführten kleineren Gletscher der M. BlancGruppe).

Im allgemeinen reagieren Gletscher mit größerem Einzugsgebiet und einer Zunge im Tal schneller bei den Vorstößen (Grand-Croux 1820, die meisten größeren Gletscher um 1860), bei günstiger Lage durch das allmähliche Ausdünnen der Zunge aber zunächst langsamer bei Rückzügen (Grand-Etrét und Vaudet ab 1920).

Verhältnismäßig breit und flach auslaufende Gletscher verändern dagegen eher ihre Breite und Dicke als ihre Länge, holen bei Rückzügen dann aber oft das zunächst Versäumte um so schneller nach, sobald die immer dünner gewordene Zunge recht plötzlich auf größeren Flächen schwindet (Laveciau und seine Nachbarn).

Hinzu kommen natürlich (außer verschiedener Höhenlage und Exposition) auch noch andere Ursachen für weitere Differenzierungen, wie Felsriegel mit ihrer Stauwirkung (Rutor im 19. Jh.), oder Sperrung des Abfließens durch einen weiter unten ins Tal vorgestoßenen Gletscher (Ep. und Va. mehrmals vor allem im SW-Teil des M. Blanc-Gebietes und um Cogne; die beiden etwa gleich großen Triolet und Pré-de-Bar flossen aber nebeneinander her), oder gar Bergstürze (Brenva, wohl auch Triolet).

All das sollte man ja eigentlich als selbstverständlich ansehen, doch scheint vor allem SACCO solche Regeln nicht zu kennen. Er nennt z. B. alle prähistorischen Moränen innerhalb der Seitentäler erster Ordnung und im Valdigna, wohl nach Novarese 1915, einfach „dauniano“. Wirklich genaue und überlegte Durchverfolgung (an Stelle von „schematicamente") hätte bei der umfangreichen und jahrzehntelangen Lokalkenntnis von S ja schließlich zur klaren Unterscheidung von Va. und Ep. (wenn auch wohl unter anderen Lokalnamen) führen müssen. Schließlich unterscheiden sich die entsprechenden Endmoränen ja meist schon in ihrer Form (s. u.). Als einziger der älteren Bearbeiter erkannte Peretti (1937) im Verney-Einzugsgebiet westlich la Thuile Moränen, die zum Va. gehören dürften (sein „Stadium $\varepsilon$ ?"). Beschel 1958 bezeichnet ähnliche Moränen leider mit dem hier völlig verfehlten Ausdruck „Larstig“ (für dieses Stadium lassen sich im Untersuchungsgebiet keinerlei Anzeichen finden), oder er schließt sich $\mathrm{S}_{\mathrm{ACCO}}$ an.

\section{Die Moränen}

Die Moränen von 1920 sind als jüngste (Ausnahmen: Pré-de-Bar mit 1935, 1929, 1926 und 1920, Triolet mit 1929, 1926 und 1920, Brenva mit 1940 statt 1920 und einer weiteren etwa von 1950, und Tráio mit etwa 1940 und 1920) überall dort entwickelt, wo die Gletscher damals nicht gerade auf steileren Felsplatten endeten. Im allgemeinen handelt es sich um niedrige, oft mehr aus einer Reihe größerer Blöcke bestehende Moränenzüge und um die etwa in halber Höhe an die großen historischen Seitenmoränen geklebten jüngeren Streifen.

Die Moränen von 1860 gibt es an allen Gletschern zumindest stückweise. Auch auf Felsplatten sind sie, wenn diese nicht gerade sehr steil sind, wenigstens angedeutet. Auf 
den nur relativ wenig geneigten Felsplatten des Paradiso-Gebietes werden sie bis über $60 \mathrm{~m}$ hoch. Die Innenseiten der Moränen sind überall noch sehr frisch und unausgeglichen, z. T. sogar kanneliert. Die Kerne der hohen Seitenmoränen dürften natürlich meist älter sein. Das ist vor allem dann erkennbar, wenn die Außenseiten bereits reichlich Vegetation tragen.

Einen Sonderfall für die jüngste Zeit stellt das Verhalten des Brenva-Gletschers seinen hohen Seitenmoränen gegenüber dar (ähnlich verhielt sich zu Beginn des Jahrhunderts auch noch der Miage-Gletscher). Die meist hohen Brenva-Seitenmoränen wurden nicht nur durch den Bergsturz von 1920, sondern in den darauf folgenden Jahren auch noch vom Gletscher aus durch Material der Obermoräne frisch überschüttet. Teilweise wurden sie sogar durch die vorstoßende Gletscherzunge gekappt (vgl. VALBUSA 1927). Ahnlich muß man sich wohl die allmähliche Entwicklung der im allgemeinen mächtigen Seitenmoränen der Gletscher in historischer Zeit vorstellen.

Die Moränen von 1820 sind nur bei den größten Gletschern klar von den anderen $\mathrm{zu}$ unterscheiden. Sonst findet man vor allem vorderste Teile der Seitenmoränen aus dieser Zeit, seltener auch Stücke der Endmoränen. Oft mag der Stand von 1820 dem von 1860 geglichen haben und deshalb überhaupt keine eigene Moräne ausgebildet worden sein.

Der Lauson- und der Rutor-Gletscher weisen aus dem 19. Jh. drei, statt sonst zwei, Endmoränen auf.

Eindeutig noch $\ddot{1} 1 \mathrm{te}$ re, oft auch etwas verwaschenere und meist durch die dichtere Vegetation darauf schon von weitem als älter erkennbare Mor änen a us histori$\mathrm{s} \mathrm{ch}$ e $\mathrm{r} \mathrm{Z}$ e it lassen sich in vielen Fällen feststellen (Beschel 1958 datiert diejenigen des Paradiso-Hauptgebietes), sonst scheinen die entsprechenden Gletscherstände gleich oder kleiner als der größte des 19. Jh. gewesen zu sein. Einige untere Teile der Außenseiten hoher Seitenmoränen verschiedener Gletscher tragen stellenweise eine ähnlich dichte Vegetation wie diese älteren, historischen Moränen. Große Teile dieser Seitenmoränen, bei allen anderen zumindest die Kerne, dürften also auch schon aus der Zeit vom 16. oder 17. Jh. an stammen.

Bei allen historischen Moränen fällt auf, daß die Seitenmoränen im allgemeinen sehr deutlich und grobblockig entwickelt, die Endmoränen dagegen oft nur angedeutet sind. Das scheint mit der Entstehungsweise zusammenzuhängen.

Die Seitenmoränen enthalten, vor allem auf durch Felswände überragten Seiten von Gletschern, sehr viel ursprünglich auf das Eis daraufgefallenen Grobschutt. Bis zu den Endmoränen gelangen, außer bei ausnahmsweise stark schuttbedeckten Gletschern (Miage, Brenva, Triolet), dagegen nur wenige Blöcke aus der im allgemeinen nur schütteren Obermoräne. Der meist feine bis sehr feine Abschurf und Abschliff der Grundmoräne schwimmt ja weitgehend mit der "Gletschermilch" davon.

Auffällig ist übrigens auch, daß immer wieder Seiten- und selbst kleinere Endmoränen direkt auf Felsplattenrändern entlangziehen. Sollte da außer anderen Gründen auch die von den Felswänden darunter aufsteigende Warmluft eine Rolle gespielt haben? Außer in N-Exposition wäre dies ja immerhin denkbar (linke Seitenmoräne des GranParadiso-Gletschers, einige Moränen im Peradzà-Gebiet und im Val di Rhêmes).

Die Moränen des Valnontey-Standes unterscheiden sich in ihrer Vegetationsdecke überhaupt nicht mehr von der gleichzeitig eisfrei gewesenen Umgebung, ihre Form wirkt aber meist noch frisch und klar (End-, Seiten- und Mittelmoränen im Valnontey, die Moräne von Courmayeur-Villette und die gemeinsame Endmoräne von Triolet und Pré-de-Bar). Im Val Veni scheint der Kern der heutigen hohen Seitenmoränen (Miage vor allem) jeweils z. T. bereits aus dem Va. zu stammen. Vielleicht spielen dort selbst noch Reste der Mittelmoränen aus dem Ep. eine gewisse Rolle, sie könnten später sozusagen als Leitschienen gedient haben. 
Die Moränen des E pine 1-Standes sehen dann oft wesentlich anders aus. Seitenmoränen als selbständige Stücke sind kaum erhalten (eher schon Eisrandterrassen, z. B. oberhalb Epinel), dafür aber Endmoränen von z. T. recht seltsamem Habitus. Alle wirklich großen Reste von Ep.-Endmoränen gleichen nämlich in ihrer heutigen Form eher ziemlich steilen Schwemmkegeln, doch liegen sie jeweils erhöht $\mathrm{z}$ w is c h e $\mathrm{n}$ zwei Bächen, können von diesen also nicht gebildet worden sein. Nur die kleineren Wälle (entstanden durch Aufspaltung in mehrere, hintereinander liegende Einzelwälle in oft kilometerweitem Abstand) sind als deutliche Endmoränen, den Va.-Moränen ähnlich, entwickelt. Oberhalb der oft in Talweitungen gelegenen größeren Ep.-Endmoränen tritt meist auch eine gewisse Terrassierung der jungen Talfüllung auf.

Einen Sonderfall stellt die fast $2 \mathrm{~km}$ lange, mächtige Talfüllung im Gebiet der „Piano di Sylvenoire" zwischen Lillaz und Cogne dar. Vergleicht man diese Talfüllung mit den anderen Ep.-Resten, so fällt sofort die hier (erst recht im Verhältnis zur Größe des Einzugsgebietes) viel größere Materialmenge auf. Das dürfte natürlich z. T. auf die Sperrung des Abflußweges durch den damals bei Cogne noch in das Tal der Grand Éyvia reichenden Valnontey-Gletscher zurückzuführen sein, sicher ist aber auch viel älteres Material daran beteiligt. Die Streckung aller Formen im Bereich der Piano di Sylvenoire parallel zum Tal deutet ja auch darauf hin, daß es sich hier (zumindest zeitweilig) um eine Art Aufgleitbahn für das von Lillaz kommende Eis gehandelt haben dürfte.

Oberhalb davon, rund um Lillaz, entstanden beim Rückzug des Eises im ehemaligen Zungenbecken schöne Eisrandterrassen.

Das bekannte Endmoränengebiet von A y mavilles-Sarre bildet schließlich den unteren Abschluß des Untersuchungsgebietes. Das Eis reichte hier bis auf $620 \mathrm{~m} \mathrm{MH}$ herunter. Hinter einer Reihe von durch einzelne Buckel bis Wälle erkennbaren Endmoränen entwickelten sich in der Talfüllung nach Rückzug des Eises schöne, verschieden stark eingesenkte Mäander der Dora Báltea. Heute ist der Fluß bis $50 \mathrm{~m}$ in die oberste, vor allem auf der N-Seite erhaltene Terrassenfläche eingesenkt, deren Oberkante auf die damals tiefste Stelle der ehemaligen Endmoräne eingestellt ist.

\section{Die Datierung der prähistorischen Gletscherstände}

Schwer zu beantworten ist die Frage nach der Datierung oder besser nach der Parallelisierung der verschiedenen prähistorischen Gletscherstände im Untersuchungsgebiet mit den bekannten Gletscherständen im Bereich der Ostalpen. Im allgemeinen wird hierbei die Schneegrenzdepression benutzt (über die rezente Schneegrenze im Untersuchungsgebiet vgl. Peretri 1931). Nimmt man als vereinfachtes Maß für die Schneegrenzdepression im Untersuchungsgebiet die Hälfte des VD-Wertes, so ergibt sich im Vergleich die folgende Übersicht:

\begin{tabular}{|c|c|c|c|}
\hline $\cos _{2}$ & $\begin{array}{l}\text { ZIENERT } 1965 \\
\text { "korrigierter“ } \\
\text { Wert }\end{array}$ & $\begin{array}{l}\text { Penetti } \\
1937 \\
\text { (Verney) }\end{array}$ & $\begin{array}{l}\text { KLEBELSBERG } \\
1948 \\
\text { (Ostalpen) }\end{array}$ \\
\hline $\begin{array}{l}1952 \\
1930\end{array}$ & $\begin{array}{l}-35 \mathrm{~m} \\
\text { Berechnungsbasis }\end{array}$ & & \\
\hline 1920 & $35 \mathrm{~m}$ & --- & ? \\
\hline 1860 & $65 \mathrm{~m}$ & & \\
\hline 1820 & $130 \mathrm{~m}$ & $70-120 \mathrm{~m}$ & $50-80 \mathrm{~m}$ \\
\hline 17/18. Jh. & $140 \mathrm{~m}$ & --- & \\
\hline Va. & $215 \mathrm{~m}$ & $\varepsilon \quad 230 \mathrm{~m}$ & $100-120 \mathrm{~m}$ Egesen \\
\hline Ep. & $515 \mathrm{~m}$ & $\delta$ & 300-400 m Daun \\
\hline Ay. 1) & $970 \mathrm{~m}$ & $\gamma$ & $600 \mathrm{~m}$ Gschnitz \\
\hline Fénis & $\left.1020 \mathrm{~m}^{2}\right)$ & $\beta$ & 然 \\
\hline Chatillon & $\left.1050 \mathrm{~m}^{2}\right)$ & $\alpha$ & \\
\hline Ivrea min. & $\left.1180 \mathrm{~m}^{2}\right)$ & - & $1200 \mathrm{~m}$ Würm-Maximum \\
\hline
\end{tabular}


Vergleicht man die verschiedenen Werte der Tabelle, so ergeben sich im Untersuchungsgebiet bereits für die historischen Gletscherstände wesentlich höhere Werte für die Schneegrenzdepression als in den Ostalpen. Noch größer wird die Differenz dann bis zum Ep., das man, den italienischen Autoren entsprechend, wohl dem Daun gleichstellen muß, denn dazwischen fehlen jegliche Endmoränen außer dem Va., welches wieder für die Gleichstellung mit Daun zu geringe Depressionswerte aufweisen würde. Ahnlich verhält es sich mit Aymavilles und Gschnitz. Bei den noch tieferen und älteren, schon außerhalb des Untersuchungsgebietes gelegenen Rückzugsstadien gleichen sich die Depressionswerte allmählich wieder denjenigen der Ostalpen an. Welche Gründe kommen für dieses Verhalten in Frage? Die Hauptrolle dürfte die viel größere Reliefenergie im Untersuchungsgebiet spielen (M. Blanc $4810 \mathrm{~m}$, Würm-Maximum bei Ivrea etwa $200 \mathrm{~m} \mathrm{MH}$ ). Die Gletscher konnten dadurch relativ schnell recht geringe Meereshöhen erreichen, und das ergibt natürlich viel höhere Werte bei der Berechnung der Schneegrenzdepression. Mangels eines besseren Anhaltspunktes muß man aber, trotz all ihrer Problematik, auf sie zurückgreifen. Natürlich dürften auch klimatische Gründe mitgespielt haben, sie lassen sich aber nicht im einzelnen definieren. Trotz der z. T. wesentlich höheren Depressionswerte ergeben sich aber in anderer Beziehung viele Ahnlichkeiten mit den Ostalpen (vgl. am kürzesten Klebelsberg: Handbuch der Gletscherkunde und Glazialgeologie I, 1948, S. 706 f.). Die Ep.-Endmoränen liegen nämlich in vergleichbaren Tälern hier, wie dort die Daunmoränen, in 1400-1600 m MH, und die Ay.-Endmoränen hier treten, wie dort die Gschnitzmoränen, etwa am Ausgang der Seitentäler 1. Ordnung in das Haupttal auf (das Valdigna kann man hier ruhig als Seitental 1. Ordnung ansehen).

Aus dem Vorstehenden ergibt sich schließlich, daß das bisher von den Italienern "Courmayeur" genannte und dem Daun gleichgesetzte Stadium zur Vermeidung von Mißverständnissen umbenannt werden muß. Zumindest wären „Courmayeur-Verrand “ und „Courmayeur-Villette“ zu unterscheiden. Um wirklich klare Verhältnisse zu schaffen, möchte ich aber vorschlagen, statt dieser Doppelnamen die beiden in der vorliegenden Untersuchung benutzten Bezeichnungen „Epinel“ und „Valnontey“ als Lokalnamen zu benutzen.

Es ergäbe sich dann innerhalb des Untersuchungsgebietes folgende Gliederung der "stadi postwürmiani“ (vgl. vor allem Novarese 1915 und als bisher einzigen für „ $\varepsilon^{\text {" }}$ Peretti 1937):

$$
\begin{array}{ll}
\gamma & \text { Aymavilles - Gschnitz } \\
\delta & \text { Epinel - Daun } \\
\varepsilon & \text { Valnontey - Egesen. }
\end{array}
$$

\section{Entwicklungs-Überblick}

Die ältesten Moränen innerhalb des Untersuchungsgebietes stammen aus dem Gschnitz-Aymavilles. Damals waren noch alle größeren Gletscher des Untersuchungsgebietes verbunden, endeten aber nur wenig unterhalb ihres Zusammenflusses. Die entsprechenden Endmoränen sind noch heute deutlich und z. T. als mächtige Bogenstücke erhalten. Spätestens gleichzeitig wurde das selbst wesentlich ältere Konfluenzbecken westlich dieser Moränen, noch unter der immer mehr an Transportkraft verlierenden Gletscherzunge, teilweise verschüttet.

Während des Rückzuges der Gletscher in die südlichen Seitentäler und in das Valdigina hinein füllte sich das freiwerdende Zungenbecken hinter der Gschnitz-Aymavilles-Endmoräne auch noch mit glazifluvialen Ablagerungen. Bei deren und des Moränenmaterials Zerschneidung entstanden die Terrassen bis um Villeneuve. 
Bis zum D a u - E p in e 1 war der vorher gerade noch einheitliche, recht große Eisstrom längst in eine ganze Anzahl einzelner Talgletscher zerfallen (Peradzà, Bardoney, Valeille, Valnontey, Valsavaranche, Val di Rhêmes, Valgrisanche, Rutor, Val de la Lex Blanche mit Val Veni, und Triolet mit Pré-de-Bar, um nur die wichtigsten zu nennen). Die fünf größten hatten etwa die Länge des heutigen Aletschgletschers, vier davon endeten jeweils etwa in der Mitte der südlichen Seitentäler.

Viele der kleineren, seitlichen Zuflüsse dürften die Oberfläche der Talgletscher nur gerade noch erreicht haben. Sie schützten dadurch aber immer wieder stückweise (und zeitweilig) die im Tal liegenden Gletscherzungen gegen oberflächliches Abschmelzen und trugen dadurch doch etwas zur längeren Erhaltung der Grundsubstanz dieser Zungen bei. Natürlich gab es daneben auch viele völlig isolierte, kleinere Gletscher.

Dann zerfiel ein Teil der Talgletscher weiter, vor allem der vorher zumindest optisch einheitlich gewesene Gletscher im Val de la Lex Blanche und Val Veni. Alle anderen gingen natürlich ebenfalls zurück.

Letztmals hielten die Gletscherenden, wenn auch wohl nur wesentlich kürzer als in den vorhergegangenen Zeiten, im Eg e s e n-Valnon te y. Nur in schmalen und auf allen Seiten von großen, hochgelegenen Firnmulden eingerahmten Tälern blieben während dieser Zeit noch größere Talgletscher erhalten, die anderen Gletscher waren im allgemeinen nicht wesentlich größer als später während des Höchststandes in historischer Zeit.

Wieweit die Gletscher während der postglazialen Wärmezeit zurückgingen und wie viele dabei völlig verschwanden, läßt sich leider nicht feststellen.

Vom 15. Jh. an folgte jedenfalls, wohl gleichzeitig mit der Neubildung vieler kleinerer Gletscher, der historische Gletschervorstoß. Am schnellsten konnten natürlich solche Gletscher reagieren, die noch einen gewissen Eisbestand aufzuweisen hatten. Wohl auch deshalb erreichten z. B. die größeren M. Blanc-Gletscher ihr Maximum bereits im 17. Jh., während viele andere damals noch etwas nachhinkten.

Die Endmoränen der ersten Vorstöße wurden bei später folgenden, größeren Vorstößen überrannt, die Seitenmoränen immer stärker erhöht.

Einige Gletscher stießen sogar wieder aus kleineren Seitentälern in die größeren Täler vor und überfuhren dabei Almen (Triolet) oder gar von Feldern umgebene Siedlungen (Brenva).

Manche Gletscher sperrten durch ihre Zungen den Abfluß der Schmelzwässer aus der Umgebung ab. So kam es an mehreren Stellen (Combal/Miage, Pré-de-Bar/Triolet, Rutor) zur Bildung von Gletscherstauseen und den dann üblichen, wiederholten Ausbrüchen mit z. T. verheerenden Folgen.

Der Vorstoß mit dem Maximum 1818-1820 scheint bei vielen mittleren Gletschern der größte in historischer Zeit gewesen zu sein.

Die Moränen von 1846-1864 (das Maximum lag bei den größeren Gletschern meist wesentlich früher als bei den kleineren) sind bei allen Gletschern zumindest stückweise zu finden und im allgemeinen deutlich. Die Innenseiten vor allem der hohen Seitenmoränen aus dieser Zeit sind oft noch übersteil und deshalb recht beweglich.

Nach stärkerem Rückzug erfolgte schließlich der letzte allgemeine, wenn auch meist bescheidene Vorstoß bis um 1920. Nur wenige Gletscher (Tráio, Brenva, Triolet und Préde-Bar) weisen deutliche noch jüngere Moränen auf (von ganz kleinen "Jahres-Moränen“, von den Italienern „morene da contrazione“ genannt, abgesehen).

Dann folgte der zur Zeit immer noch andauernde Rückzug und damit der Zerfall einiger der in historischer Zeit neu entwickelten Talgletscher, vor allem in den Talschlüssen. Viele kleinere Gletscher verschwanden völlig. 
Seit 1954 scheint sich der Rückzug (wohl nur vorübergehend) zu verlangsamen. 1960 und 1963/64 z. B. wurden sogar einige Gletscher des Paradiso-Gebietes durch Firnschnee nach unten verlängert, statt weiter zurückzuschmelzen.

\section{$\mathrm{Sch}$ if t t u m}

\section{(Zitiert wird nur Literatur aus dem Untersuchungsgebiet.)}

Baretti, M.: I ghiacciai antichi e moderni. - Diss. Torino 1866 (zitiert nach Novarese 1915). - Il lago del Rutor (alpi graje settentrionali). - Boll. CAI 14, S. 43-95, 1880 (=1880 a). - Il ghiacciaio del Miage (versante italiano del Monte Bianco). - Mem. Acc. Scienze Torino 32/2, S. 269-302, 1880 (=1880 b). - - Geologia della provincia di Torino. Torino 1893.

Beschel, R.: Flechten als Altersmaßstab rezenter Moränen. - Z. Gletscherk. Glazialgeol. 1, S. 152161, 1950. - - Lichenometrie im Gletscher-Vorfeld. - Jb. Ver. Schutze Alpenfl. etc. 10, S. 164-185, 1957. - - Ricerche lichenometriche sulle morene del gruppo del Gran Paradiso. - N. Giorn. bot. ital. 65, S. 538-591, 1958.

Bossolasco, M.: Studio topografico e stato attuale del ghiacciaio del Rutor. - Boll. Comit. glac. ital. 8, S. 23-31, 1928. - - Sulle condizioni attuali di alcuni ghiacciai del gruppo del Gran Paradiso. - Boll. Gomit. glac. ital. 9, S. 109-143, 1929.

CAPELlo, C. F.: Ricerche glaciologiche e morfologiche sul gruppo Levanne-Carro-Baséi (bacino idrografico dell'Orco). - Boll. Comit. glac. ital. 9, S. 67-107, 1929. - - Aspetti della glaciazione sul versante italiano del massiccio del Monte Bianco. - L'Universo 17, S. $897-$ 917, 1936 (=1936 a). - - La glaciazione attuale nel massiccio del Monte Bianco. Caratteri morfologici e morfometrici dei ghiacciai sul versante italiano. - Boll. Comit. glac. ital. 16, S. 154-224, 1936 (=1936 b). - - Sui terrazzi fluvio-glaciali e sulla morfologia carsica delle valli di Pré-St-Didier. - Boll. Comit. glac. ital. 19, S. 135-177, 1939. - Rilievi sui ghiacciai Pré-de-Bar, Allée-Blanche e Triolet nel decennio 1929-1939. - Boll. Comit. glac. ital. 20, S. 85-115, 1940. - - Studio sul ghiacciaio della Brenva. - Boll. Comit. glac. ital. 21, S. 129-153, 1941. - - Sulla genesi delle morene da contrazione. Boll. Comit. glac. ital. 23, S. 29-54, 1945. - - Morfologia e morfometria dei ghiacciai della valle di Rhêmes. - Riv. mensile CAI 67, S. 22-43, 1948. - - Le pietraie semoventi nel gruppo del Gran Paradiso. - Riv. mensile CAI 78, S. 294-300, 371-376, 1959 (=1959 a). - - Frane-valanghe di ghiaccio nel gruppo del Monte Bianco. - Boll. Comit. glac. ital. II/8/1, S. 125-138, 1959 (=1959 b).

Снabod, R.: Il plastico della valle d'Aosta di Alessio Nebbia. - Riv. mensile CAI 76, S. 165-168, 1957.

Commissione del parco nazionale del Gran Paradiso: Il parco nazionale del Gran Paradiso. Torino 1925 (später noch mehrfach aufgelegt).

Dal Piaz, G. B.: Geologia della catena Herbetet - Grivola - Grand Nomenon. - Mem. Ist. geol. Univ. Padova VII/6, 1928.

Druetri, A.: Ricerche sui fenomini glaciali nel gruppo del Gran Paradiso. Campagna glaciologica del 1896. - Boll. CAI 20, S. 297-320, 1897.

Druetti, A. \& Porro, F.: Osservazioni sui ghiacciai del gruppo del Gran Paradiso. Contributo allo studio del movimento dei ghiacciai. - Boll. CAI 19, S. 145-180, 1896.

Giannitrapani, L.: La valle d'Aosta, monografia geografica. - L'Universo 14, S. 233-266, 321-360, 413-443, 509-553, 1933.

Gignoux, M.: Ce que le glacier de la Brenva a appris aux géologues. - Rev. Valdôtaine 1, S. 88-95, 1948..

Hermann, F.: Sulla geologia della valle di Rhêmes. - Boll. Soc. geol. ital. 41, S. 214-220, 1922.

KInZL, H.: Die größten nacheiszeitlichen Gletschervorstöße in den Schweizer Alpen und in der Mont-Blanc-Gruppe. - Z. Gletscherk. 20, S. 269-397, 1932.

Langini, O.: Il parco nazionale del Gran Paradiso. - L'Universo 40, S. 93-106, 1960.

Lesca, C.: Osservazioni sui laghi del ghiacciaio del Miage. - Boll. Comit. glac. ital. II/7/1, S. 29-55, 1956.

Marengo, G.: Monografia del ghiacciaio della Brenva. - Boll. CAI 15, S. 3-9, 1881.

Montr, V.: Primo saggio di nuove ricerche sulle oscillazioni dei ghiacciai del Gran Paradiso. - Riv. geogr. ital. 18, S. 46-60, 1911.

Moretri, A.: Osservazioni su alcuni ghiacciai del gruppo del Gran Paradiso. - Boll. Comit. glac. ital. II/2, S. 5,7-74, 1951. - - Cenni sui ghiacciai del Gran-Neyron e del Timorion nel gruppo del Gran Paradiso. - Boll. Comit. glac. ital. II/3, S. 7-16, 1952. 
Novarese, V.: Il quaternario in Val d'Aosta e nelle valli del Canavese, parte III: Gli stadi postwürmiani. - Boll. Comit. geol. ital. 45, S. 137-196, 1915.

Peretti, L.: I ghiacciai del versante meridionale del gruppo del Gran Paradiso. - Boll. Comit. glac. ital. 10, S. 45-89, 1930. - - Il limite climatico delle nevi permanenti nelle alpi occidentali italiane. - Boll. Comit. glac. ital. 11, S. 151-187, 1931. - - Nuove osservazioni e misure al ghiacciaio del Rutor. - Boll. Comit. glac. ital. 14, S. 173-184, 1934. - - I ghiacciai del gruppo del Gran Paradiso nella valle della Grand'Eiva. - Boll. Comit. glac. ital. 15, S. 102-135, 1935 (=1935 a). - - Considerazioni sul glacialismo attuale nel gruppo del Gran Paradiso. - Boll. Comit. glac. ital. 15, S. 136-154, 1935 (=1935 b). - -Morfologia glaciale nella valle della Dora di Verney (alta valle d'Aosta). - Boll. Comit. glac. ital. 17, S. 141-159, 1937.

Peretti, L. \& Lesca, C.: Nuovi rilevamenti topografici di fronti glaciali nella Val Ferret (gruppo del Monte Bianco). - Boll. Comit. glac. ital. II/4, S. 221-229, 1953. - - Rilevamento stereofotogrammetrico dei ghiacciai nell'alta Valnontey (gruppo del Gran Paradiso). Boll. Comit. glac. ital. II/7/1, S. 13-18, 1956.

Porro, F.: Nuove osservazioni sui ghiacciai del Gran Paradiso e del M. Bianco. - Boll. CAI 36, S. 127-156, 1903. - - Primi studi topografici sul ghiacciaio del Miage. - Boll. Comit. glac. ital. 1, S. 31-44, 1914.

Richter, F.: Geschichte der Schwankungen der Alpengletscher. - Z. DƠAV 1891, S. 1-74, 1891.

Rovereto, G.: Geomorfologia del gruppo del Gran Paradiso. - Boll. CAI 38, S. 199-271, 1906. - Forme della terra. Trattato di geologia morfologica (geomorfologia). Milano 1923-25.

SAcco, F.: Il ghiacciaio ed i laghi del Rutor. - Boll. Soc. geol. ital. 36, S. 1-33, 1917 (=1917 a). - L'apparato morenico del ghiacciaio del Miage. - Boll. Soc. geol. ital. 36, S. 323-354, 1917 (=1917 b). - - I ghiacciai italiani del gruppo del Monte Bianco. - Boll. Comit. glac. ital. 3, S. 21-102, 1919. - - Le oscillazioni glaciali. - Atti Acc. Scienze Torino 55, S. 139156, 1919/20. - - Il glacialismo nel gruppo del Monte Emilius (valle d'Aosta). - Boll. Soc. geol. ital. 39, S. 111-131, 1920. - - Il glacialismo nelle Valgrisanche, Rhêmes e Savaranche. - L'Universo 1921, S. 743-785, 1921. - - Il glacialismo nel gruppo del Gran Paradiso. - Boll. Comit. glac. ital. 4, S. 121-168, 1921 und 5, S. 52-121, 1923, $(=1921+$ 23). - - Il glacialismo nelle valli dell'Orco e delle Soana. - Boll. Comit. glac. ital. 6, S. 35-64, 1925. - - Il glacialismo nella valle d'Aosta. - Min. Lav. pubbl., Uff. idrogr. Po 1927. - - L'anfiteatro morenico recente del Rutor. - L'Universo 15, S. 907-922, 1934 (=1934 a). - - Le alpi. TCI Milano 1934 (=1934 b). - - Il glacialismo piemontese (valle d'Aosta). - L'Universo 19, S. 217-231, 337-352, 1938. - - Il quaternario nell'alta valle di Rhêmes. - Atti Acc. Scienze Torino 74, S. 86-104, 1939 (=1939 a). - - Il quaternario nel gruppo del Gran Paradiso. - Boll. Uff. geol. d'Italia 64, S. 1-34, 1939 (=1939 b). - Il quaternario nella catena del Monte Bianco. - Mem. Acc. Scienze Torino 70/2, S. 1-22, 1942.

Sestini, A.: Osservazioni e ricerche sulle valli di Courmayeur. - Mem. geol. geogr. G. Dainelli 1, S. 215-290, 1930.

Silvestri, E.: Il movimento frontale del ghiacciaio della Brenva negli ultimi cento anni dal 1818 al 1923. - Atti Acc. pont. n. Lincei 78, S. 195-200, 1925.

Socin, C.: Panorama morfologico e geologico del Piemonte. - Pubbl. Ist. geol. Univ. Torino 3 , S. 59-82, 1954.

Touring Club Italiano: L'Italia fisica. Conosci l'Italia 1, Milano 1957.

Valbusa, U.: La catastrofe del Monte Bianco e del ghiacciaio della Brenva. - Boll. Soc. geogr. ital. 58, S. 151-162, 1921. - - Il ghiacciaio della Brenva. - Riv. CAI 46, S. 273-280, 331-344, 1927. - - La prima frana valanga del Monte Bianco sul ghiacciaio della Brenva (14 nov. 1920). - Boll. Soc. geogr. ital. 68, S., 118-125, 1931.

VANNI, M.: I laghi del Nivolet (alta Valsavaranche). - Boll. Soc. geogr. ital. 68, S. 646-653, 1931. - - Le variazioni frontali dei ghiacciai italiani negli ultimi venticinque anni e l'opera del Comitato Glaciologico Italiano. - Boll. Comit. glac. ital. 25, S. 75,-85, 1948. - - Il glacialismo attuale nelle alpi piemontesi nei rapporti col regime dei corsi d'acqua. - Boll. Comit. glac. ital. II/8/1, S. 43-124, 1959.

Vanni, M., Origlia, C. \& de Gemini, F.: I ghiacciai della valle d'Aosta. - Boll. Comit. glac. ital. II/4, S. 1-175, 1953.

Virgilio, F.: Di un antico lago glaciale presso Cogne in Val d'Aosta. - Atti Acc. Scienze Torino 1886, S. 291-303, 1886.

Hinzu kommen die laufenden Vermessungsberichte im Boll. Comit. glac. ital., von deren Einzelaufführung hier abgesehen werden soll. 
Topographische Karten $1: 25000$ des Istituto Geografico Militare (IGM):

\begin{tabular}{|c|c|c|c|c|c|c|}
\hline 27 & II $\underset{S E}{N E}$ & M. Bianco & 41 & & $\mathrm{NE}$ & Rhêmes St. Georges \\
\hline 28 & IV SO & $\begin{array}{l}\text { 1a Thuile } \\
\text { la Vachey }\end{array}$ & & & SE & $\begin{array}{l}\text { Grande Sassière } \\
\text { Rhêmes Notre Dame }\end{array}$ \\
\hline & $\mathrm{SE}$ & Gran S. Bernardo & & III & NO & Granta Parei \\
\hline & III NO & Courmayeur & & & NE & Colle del Nivolet \\
\hline & $\mathrm{NE}$ & Bosses & & II & NO & Ceresole Reale \\
\hline & SO & la Salle & & & SO & Gran Paradiso \\
\hline & SE & Arvier & & & SE & Torre del Gran S. Pietro \\
\hline & II SO & Aosta & & & nO & la Grivola \\
\hline & SE & M. Emilius & & & NE & Cogne \\
\hline 41 & IV NO & Valgrisanche & 42 & IV & $\mathrm{NO}$ & Punta Tersiva \\
\hline
\end{tabular}

Außer vom zuerst und vom zuletzt genannten Blatt gibt es auch Anaglyphen-Karten etwa im selben Maßstab.

Carta turistica del Monte Bianco $1: 25000$ (IGM) in 6 Blättern.

Topographische Karten $1: 100000$ des IGM:
27 M. Bianco
41 Gran Paradiso
28 Aosta
42 Ivrea

Carta geologica d'Italia 1:100000:
27 M. Bianco
28 Aosta
41 Gran Paradiso
42 Ivrea

Carta geologica delle alpi nord-occidentali $1: 200000$ von F. Hermann, Milano 1937 (mit Erläuterungen von 1938).

Manuskr. eingeg. 28. 5. 1965.

Anschrift des Verf.: Dr. Adolf Zienert, 69 Heidelberg, Theodor-Körner-Straße 3. 